\title{
A natural fusion of flavodiiron, rubredoxin, and NADH:rubredoxin oxidoreductase domains is the highly efficient water-forming oxidase of $T$. vaginalis.
}

\author{
Evana N. Abdulaziz ${ }^{1 \Delta}$, Tristan A. Bell ${ }^{2}$, Bazlur Rashid ${ }^{3}$, Mina L. Heacock ${ }^{1}$, Owen S. Skinner ${ }^{2,4}$, \\ Mohammad A. Yaseen ${ }^{5}$, Luke H. Chao ${ }^{2}$, Vamsi K. Mootha ${ }^{2,4}$, Antonio J. Pierik ${ }^{3}$ and Valentin Cracan ${ }^{1,6^{*}}$ \\ ${ }^{1}$ Scintillon Institute, San Diego, CA 92121, USA \\ ${ }^{2}$ Department of Molecular Biology, Massachusetts General Hospital, Harvard Medical School, Boston, \\ MA 02142, USA \\ ${ }^{3}$ Faculty of Chemistry, University of Kaiserslautern, Kaiserslautern, D-67663, Germany \\ ${ }^{4}$ Howard Hughes Medical Institute, Massachusetts General Hospital, Boston, MA 02142, USA \\ ${ }^{5}$ Department of Bioengineering, Northeastern University, Boston, MA 02115, USA \\ ${ }^{6}$ Department of Chemistry, The Scripps Research Institute, La Jolla, CA 92037, USA \\ ${ }^{\Delta}$ Present position: CW Associate Scientist, Amgen, Thousand Oaks, CA, 91320
}

*corresponding author: Valentin Cracan

Tel: (858) 202-5251; E-mail: vcracan@scripps.edu

\section{Keywords}

Microaerophilic protozoan parasites, flavodiiron protein (FDP), rubredoxin, NADH:rubredoxin oxidoreductase (NROR), rubredoxin:oxygen oxidoreductase (ROO), $\mathrm{H}_{2} \mathrm{O}$-forming NADH oxidase (NOX), oxygen metabolism, nicotinamide adenine dinucleotide $\left(\mathrm{NAD}^{+}\right)$, nicotinamide adenine dinucleotide phosphate $\left(\mathrm{NADP}^{+}\right)$, cellular redox environment.

\begin{abstract}
Microaerophilic pathogens such as Giardia lamblia and Trichomonas vaginalis have robust oxygen consumption systems to detoxify oxygen and maintain the intracellular redox balance. This oxygen consumption is a result of the $\mathrm{H}_{2} \mathrm{O}$-forming NADH oxidase activity of two distinct flavin-containing systems: $\mathrm{H}_{2} \mathrm{O}$-forming NADH oxidases (NOXes) and multicomponent flavodiiron proteins (FDPs). Both systems are not membrane-bound and recycle NADH into oxidized $\mathrm{NAD}^{+}$while simultaneously removing $\mathrm{O}_{2}$ from the local environment, making them crucial for the survival of human microaerophilic pathogens. In this study, using bioinformatic and biochemical analysis, we show that $T$. vaginalis lacks a NOX-like enzyme, and instead harbors three proteins that are very close in their amino acid sequence and represent a natural fusion between $\mathrm{N}$-terminal FDP, central rubredoxin and C-terminal NADH:rubredoxin oxidoreductase domains. We demonstrate that this natural fusion protein with fully populated flavin redox centers unlike a "stand-alone" FDP (also present in T. vaginalis), directly accepts reducing equivalents of $\mathrm{NADH}$ to catalyze the four-electron reduction of $\mathrm{O}_{2}$ to water within a single polypeptide and with an extremely high turnover. Using single particle electron cryo-microscopy (cryo-EM) we present structural insight into the spatial organization of the FDP core within this multidomain fusion protein. Our studies represent an important addition to our understanding of systems that allow human protozoan parasites to maintain their optimal redox balance and survive transient exposure to oxic conditions.
\end{abstract}




\section{Introduction}

Trichomonas vaginalis is a microaerophilic human protozoan parasite that causes trichomoniasis, one of the most common sexually transmitted infections $(1,2)$. A distinct feature of $T$. vaginalis as well as other human parasites such as Giardia lamblia (syn. intestinalis, duodenalis) and Entamoeba histolytica is their ability to tolerate low oxygen concentrations and transient exposure to oxic conditions. This is remarkable as these protozoa depend on metabolic enzymes that are extremely sensitive to oxygen, such as pyruvate:ferredoxin oxidoreductase (PFOR) and FeFe-hydrogenase $(3,4)$. These organisms do not contain mitochondria, and instead harbor mitochondria-derived organelles called mitosomes or hydrogenosomes that lack a membrane-bound electron transport chain (ETC) to carry out oxidative phosphorylation (OXPHOS) (4). Moreover, it is well recognized that $T$. vaginalis consumes oxygen and that this oxygen consumption is insensitive to ETC inhibitors (such as cyanide or sodium azide) $(4,5)$. Over the years, most of this oxygen consumption was attributed to enzymes that catalyze a conversion of diatomic oxygen to two benign water molecules at the expense of reducing equivalents of $\mathrm{NAD}(\mathrm{P}) \mathrm{H}$ (the $\mathrm{H}_{2} \mathrm{O}$-forming NAD(P)H oxidase reaction) (Fig. 1A). This reaction not only serves the "oxygen scrubbing" role but at the same time maintains uninterrupted supply of oxidized $\mathrm{NAD}^{+}$to support optimal intracellular NADH/NAD ${ }^{+}$ ratio $(4,6)$.

Through convergent evolution, two different enzymatic systems evolved to catalyze the $\mathrm{H}_{2} \mathrm{O}$ forming NADH oxidase reaction in various bacteria and protozoa (Fig. 1A). The first system is an $\mathrm{H}_{2} \mathrm{O}-$ forming NADH oxidase (NOX) that belongs to the evolutionarily versatile "two dinucleotide binding domains" flavoproteins (tDBDF) superfamily (Fig. 1A) (7-9). These soluble NOXes are not related to the mammalian transmembrane ROS-producing NADPH oxidases (NOX1-5 and DUOX1-2). The active site of a typical tDBDF superfamily NOX enzyme ( $\sim 50 \mathrm{kDa}$ per monomer) consists of a single FAD cofactor and an adjacent redox-active cysteine that cycles between sulfenic acid and reduced cysteine to accomplish the four electron reduction of oxygen $(7,10,11)$. For example, in our previous study we extensively both biochemically and structurally characterized the water-forming oxidase reaction of L. brevis NOX ( $L b \mathrm{NOX})(11) . L b \mathrm{NOX}$ is an extremely efficient enzyme with strict specificity towards NADH, with $K_{\mathrm{MS}}$ for $\mathrm{O}_{2}$ and $\mathrm{NADH}$, of $\sim 2 \mu \mathrm{M}$ and $69 \pm 3 \mu \mathrm{M}$, respectively, and the turnover number of $648 \pm 28 \mathrm{~s}^{-1}$ while less than $2 \%$ of input electrons lead to the off-target $\mathrm{H}_{2} \mathrm{O}_{2}$ formation (11).

Flavodiiron proteins (FDPs) are a second system with $\mathrm{H}_{2} \mathrm{O}$-forming oxidase activity (Fig. 1A-C, Fig. 2A). Interestingly, some FDPs can also use $\mathrm{NO}$ gas as a substrate or can have mixed $\mathrm{O}_{2} / \mathrm{NO}$ specificity (Fig. 1B) (12-15). The minimal unit of a typical FDP (in the new classification Class A FDP, see below) is a $\sim 50 \mathrm{kDa}$ protein with two subdomains: an $\mathrm{N}$-terminal metallo- $\beta$-lactamase-like subdomain containing the dinuclear Fe-Fe center (diiron center); and a C-terminal subdomain, containing the FMN redox cofactor (15-17)(Fig. 2A). The genomes of human protozoan parasites T. vaginalis, G. lamblia and E. histolytica encode biochemically verified Class A FDPs (18-20). Within a monomer the distance between the oxygenbinding diiron center and the FMN plane is approximately $40 \AA$ in all reported structures of FDPs, much greater than the $\sim 15 \AA$ maximum distance that allows efficient electron transfer (21). As such, the functional unit of a "stand-alone" Class A FDP is comprised of a "head-to-tail" homodimer that positions the diiron center of one protomer adjacent to the FMN bound of its neighboring protomer $(15,17,19)$.

The FMN redox cofactor of FDP cannot directly receive electrons from NAD(P)H and requires additional protein adaptors as substrates. For example, FDPs can directly receive electrons from a rubredoxin $(\mathrm{Rb}), \mathrm{a} \sim 6 \mathrm{kDa}$ protein that contains a $\mathrm{Fe}(\mathrm{SCys})_{4}$ center and in some bacteria and Archaea is reduced by $\mathrm{NAD}(\mathrm{P}) \mathrm{H}$ :rubredoxin oxidoreductases (NRORs) or similar systems (4,22-26)(Fig. 1B). Notably, in biochemical and spectroscopic studies of previously characterized Class A FDPs, only artificial protein substrates were used (i.e. recombinant NADH:flavorubredoxin oxidoreductase and a truncated rubredoxin domain of flavorubredoxin, both from $E$. coli) as in the corresponding organisms $\mathrm{Rb}$ and/or NROR homologs were not identified $(16,27,28)$.

Recent bioinformatic analysis revealed that in many organisms FDPs are naturally fused to various domains, and led to the current classification of this large group of proteins (27-29). According to this classification "stand-alone" FDPs that contain only diiron and FMN centers are designated as a Class A 
FDPs or FDPAs (27) (Fig. 2A). Several additional classes were proposed for proteins in which one or more other domains are fused genetically to the C-terminal side of the FDP core (Classes B to H) (27). These additional redox centers may play crucial roles in dedicated electron transfer (ET) pathways, via which reducing equivalents of $\mathrm{NAD}(\mathrm{P}) \mathrm{H}$ or other substrates are relayed to the gas-binding diiron site (27).

The first attempt to purify the $\mathrm{H}_{2} \mathrm{O}$-forming $\mathrm{NADH}$ oxidase activity from $T$. vaginalis isolated a preparation that was active only with NADH (no activity with NADPH was detected) with a specific activity of $16.5 \mu \mathrm{mol} \mathrm{min}^{-1} \mathrm{mg}^{-1}$ and $K_{\mathrm{M}}$ for NADH of $7.4 \mu \mathrm{M}$ (30). The authors reported that the preparation consumed NADH and $\mathrm{O}_{2}$ with 2:1 stoichiometry and did not produce $\mathrm{H}_{2} \mathrm{O}_{2}$ as a byproduct. Unfortunately, neither the $\mathrm{N}$-terminal sequence nor the molecular mass of the enzyme was reported at that time. In a later study, a $\mathrm{H}_{2} \mathrm{O}$-forming NADH oxidase activity from $T$. vaginalis was purified, and exhibited a $\mathrm{V}_{\max }$ of 470

$\mathrm{s}^{-1}$ and $K_{\mathrm{M}}$ for NADH of $5.4 \mu \mathrm{M}(31)$. The authors reported that when analyzed by SDS-PAGE, the final protein preparation migrated as two closely spaced unusually high molecular mass bands of 97-97.5 kDa. The latter observation is inconsistent with NOXes being $\sim 50 \mathrm{kDa}$ proteins (see above) (30-33).

In our studies we turned our attention to the NOX-like activity ascribed to T. vaginalis because we were interested in oxygen metabolizing systems that allow unicellular organisms that lack ETC to maintain an optimal redox environment (11). To our surprise, analysis of the available genomic sequences of $T$. vaginalis showed that this organism does not contain a nox gene encoding a typical tDBDF superfamily member, but instead contains 3 copies of a natural fusion between flavodiiron, rubredoxin and NADH:rubredoxin oxidoreductase domains (Class F FDP or FDPF according to the new classification) (Fig. 2B) (27). These copies have pairwise amino acid sequence identities of 75-80\% and likely arose through gene duplication, as it is common in many protozoa (34). We subsequently overexpressed and purified these three Class F FDPs from T. vaginalis (TvFDPF 1-3). After preliminary kinetic characterization of these 3 proteins, we focused our extensive biochemical and biophysical characterization on TvFDPF3, as it was the most active and well-behaved. Our biochemical experiments with $T v$ FDPF3 confirmed that this natural fusion protein allows the crosstalk of all three domains, ultimately relaying electrons from $\mathrm{NAD}(\mathrm{P}) \mathrm{H}$ to $\mathrm{O}_{2}$. We also used single-particle electron cryo-microscopy (cryo-EM) to visualize the dimerization interface of this Class F FDP. Overall, these studies allow us to conclude that the main $\mathrm{H}_{2} \mathrm{O}$ forming oxidase activity in T. vaginalis is catalyzed by a fusion Class F FDP we identified and characterized. Our observations suggest the activity described by Bradley and Linstead in 1988, and by Tanabe in 1979, was misassigned as a NOX-like enzyme, and in fact is a Class F FDP that catalyzes the $\mathrm{H}_{2} \mathrm{O}$-forming oxidase reaction within a single polypeptide. Our spectroscopic and structural studies provide important new insight into how the Class F FDP system from $T$. vaginalis achieves extremely efficient $\mathrm{H}_{2} \mathrm{O}$-forming oxidase activity using four separate redox centers.

\section{Results}

\section{Identification of Class $\mathrm{F}$ flavodiiron proteins in $T$. vaginalis as enzyme candidates for previously reported $\mathrm{H}_{2} \mathrm{O}$-forming $\mathrm{NADH}$ oxidase activity.}

Because we were interested in evolutionary innovations that allow lower organisms that lack a membrane bound ETC to maintain an optimal intracellular redox environment we explored NOXes ascribed to human protozoan parasites (4). While we easily identified previously characterized NOX in the genome of $G$. lamblia $(32,33,35)$, the closest matches in the genome of $T$. vaginalis were three proteins, all of which were 871 amino acids in length and annotated in the GeneBank database as pyridine nucleotide-disulfide oxidoreductases or apoptosis inducing factor (TVAG_263800, TVAG_049830 and TVAG_121610) (Supplementary Table S1). On closer inspection, we noticed that these 3 proteins represent a natural fusion composed of an $\mathrm{N}$-terminal portion homologous to a flavodiiron protein, a middle rubredoxin $(\mathrm{Rb})$ domain, and a C-terminal portion that is homologous to an NADH:rubredoxin oxidoreductase (NROR) (Fig. 2B). Based on the recent classification all 3 fusion proteins in $T$. vaginalis are Class F FDPs (from now on we denote them as $T v$ FDPF1-3)(27) (Fig. 2B). 
When compared to FDPAs from T. vaginalis, G. lamblia and several other organisms, the Nterminal FDP domain of $T \nu$ FDPF1-3 includes the previously recognized canonical sequences containing the ligands of the diiron center: Fe 1 (His82-X-Glu84-X-Asp86-His87, TvFDPF3 numbering) and Fe 2 (His 148- $\mathrm{X}_{18}$-Asp166- $\mathrm{X}_{64}$-His230) (Supplementary Figure S1). The middle domain of TvFDPF1-3 is homologous to rubredoxins and the C-terminal domain of bacterial rubrerythrin (Supplementary Figure S2). A central feature of the middle domain of TvFDPF1-3 is the presence of two pairs of Cys- $\mathrm{X}_{2}-\mathrm{Cys}$ sequence patterns that coordinate the iron of the $\mathrm{Fe}(\mathrm{SCys})_{4}$ center. This middle $\mathrm{Rb}$-like domain is flanked at both sides by $\sim 25-30$ amino acid linkers.

NADH:rubredoxin oxidoreductases and NOXes both belong to the tDBDF superfamily of flavoenzymes allowing our initial BLAST analysis to identify TvFDPF1-3 (i.e. the C-terminal domain of Class F FDPs [i.e. NROR] is homologous to NOX) (Supplementary Figure S3) (9). Both the GxGxxG dinucleotide-binding motif and the NAD(P)H substrate specificity loop are identifiable in the C-terminal NROR domain of TvFDPF1-3 as well as in other closely related members of the tDBDF superfamily, including a "stand-alone" NROR, "stand-alone" NADH:ferredoxin oxidoreductase, and CoA-disulfide reductase (Supplementary Figure S3).

\section{Cloning and initial purification of Class $\mathrm{F}$ fusion flavodiiron proteins from $T$, vaginalis (TvFDPF1-} 3).

To test whether $T v$ FDPF1-3 exhibit the $\mathrm{H}_{2} \mathrm{O}$-forming oxidase activity and accept electrons directly from NADH or NADPH, the corresponding genes from $T$. vaginalis were cloned with a C-terminal HexaHis tag into a bacterial expression vector. All 3 genes had no introns, as it is common in T. vaginalis, $G$. lamblia and other parasitic protozoa. When purified, all three recombinant proteins were brown in color and migrated as $\sim 100 \mathrm{kDa}$ band as judged by the SDS-PAGE analysis (Supplementary Figure S4A-C, Supplementary Figure S5). Notably, the TvFDPF2 protein was prone to severe aggregation and during purification eluted in the void volume of the size-exclusion column. Based on analytical gel-filtration and assuming a globular shape, we estimated an apparent molecular weight for TvFDPF1 of $302 \pm 10 \mathrm{kDa}$ and for $T v$ FDPF3 of $326 \pm 5 \mathrm{kDa}$. The absorption spectra of all three oxidized proteins had clear features of flavin bands (350-500 nm) and a Fe(SCys) $)_{4}$ center of rubredoxin $(>500 \mathrm{~nm})$ at 379, 454, 475 and $568 \mathrm{~nm}$ for $T v$ FDPF1; 374, 451, 478 and $565 \mathrm{~nm}$ for $T v$ FDPF2; and 378, 451, 475 and $574 \mathrm{~nm}$ for $T v$ FDPF3 (Supplementary Figure S4B-C, Supplementary Figure S5). In the presence of excess sodium dithionite these cofactors were reduced, and the visible absorbance was almost completely bleached.

We next employed liquid chromatography-mass spectrometry (LC-MS) to determine both the molecular identity and quantity of flavin cofactors bound to $T v$ FDPF1-3. While we unambiguously identified both FAD and FMN within TvFDPF1-3, the actual stoichiometries of these cofactors per protein monomer were drastically different (Table 1). Both $T v$ FDPF1 and $T v$ FDPF3 had full occupancy with FAD per monomer $(1.02 \pm 0.05$ and $1.00 \pm 0.12$, respectively). However, FMN was present only at $0.010 \pm 0.004$ occupancy per monomer $(\sim 1 \%)$ in $T \nu$ FDPF1 and $0.090 \pm 0.006(\sim 9 \%)$ in $T \nu$ FDPF3 (Table 1). $T \nu$ FDPF2 had poor occupancy of both FAD and FMN, which is not surprising given its poor behavior during purification. Thus far only the FMN cofactor was reported in Class A FDPs, and it seemed logical that poor occupancy of FMN in our preparations of TvFDPF1-3 reflects occupancy of the N-terminal domain only. Direct addition of $300 \mu \mathrm{M}$ FMN to the lysis buffer during purification of $T \nu$ FDPF1 did not improve FMN occupancy (Table 1).

Because $\mathrm{Fe}$ is a part of both the diiron center and the rubredoxin-like domain, we measured iron content of all three proteins (Supplementary Table S2). We found that despite addition of Mohr's salt (ammonium iron(II) sulfate) during protein expression, all purified TvFDPF1-3 had substoichiometric iron occupancy ( $3 \mathrm{Fe}$ per monomer is expected). The highest Fe occupancy of $0.82 \pm 0.17$ was observed for $T v$ FDPF1, while $T v$ FDPF2 and $T v$ FDPF3 had $0.41 \pm 0.02$ and $0.58 \pm 0.10$, respectively (Supplementary Table S2). 


\section{Specific activity and specificity towards NADH and NADPH.}

Because previously reported $\mathrm{H}_{2} \mathrm{O}$-forming NADH oxidase-like activities purified from $T$. vaginalis were tested with both NADH and NADPH, we tested consumption of both redox cofactors in our enzymatic assays (Table 2, Supplementary Figure S4D-F). Michaelis-Menten analysis of the reaction catalyzed by TvFDPF1-3 indicates that NADH is the preferred substrate over NADPH for all three enzymes. The highest $V_{\max }$ of $12 \pm 1 \mu \mathrm{mol} \mathrm{min} \mathrm{mg}^{-1}$ at $37^{\circ} \mathrm{C}$ was observed for $T \nu \mathrm{FDPF} 3$. We found that the lowest $K_{\mathrm{M}}$ for NADH among all variants was $2.9 \pm 0.8 \mu \mathrm{M}$ for $T \nu \mathrm{FDPF} 1$. This value is in sharp contrast to the $K_{\mathrm{M}}$ for NADH of $T \nu \mathrm{FDPF} 3$ that was $40 \pm 8 \mu \mathrm{M}$. However, because $V_{\max }$ for $T \nu$ FDPF1 was low, the overall catalytic efficiency $k_{\text {cat }} / K_{\mathrm{M}}$ of both $T \nu$ FDPF1 and $T v$ FDPF3 as well of $T v$ FDPF2 with NADH were very similar. MichaelisMenten fitting of the NADPH data results in $K_{\mathrm{M}}$ values in the $\mathrm{mM}$ range, far above its physiological concentration (Table 2). Our results clearly support our prediction that the fusion Class F FDP from $T$. vaginalis is a "self-sufficient" enzyme that directly accepts reducing equivalents from $\mathrm{NAD}(\mathrm{P}) \mathrm{H}$. Because $T \nu$ FDPF2 had low yield and was not stable, we focused further kinetic studies on $T \nu$ FDPF1 and $T v$ FDPF3. We also determined that $T v$ FDPF3 had little activity with $\mathrm{NO}\left(<2 \%\right.$ when compared to the NADH to $\mathrm{O}_{2}$ activity, Supplementary Figure S6).

\section{Kinetics of oxygen consumption and $\mathrm{H}_{2} \mathrm{O}_{2}$ production.}

We next studied the reaction catalyzed by $T \nu \mathrm{FDPF} 1$ and 3, by monitoring both NADH and oxygen consumption simultaneously (Supplementary Figure S7A-B). To our surprise there was a clear difference in NADH-to- $\mathrm{O}_{2}$ stoichiometry for $T v$ FDPF1 when compared to $T v$ FDPF3. With $T v$ FDPF3, two consecutive additions of $250 \mu \mathrm{M}$ NADH were needed to completely reduce $\mathrm{O}_{2}$ of air-saturated buffer $(\sim 250 \mu \mathrm{M})$ to water. In contrast, $\mathrm{O}_{2}$ and NADH consumption by $T v$ FDPF1 had a 1:1 stoichiometry. The observed reaction traces support a $\mathrm{H}_{2} \mathrm{O}$-forming reaction for $T \nu \mathrm{FDPF} 3$ but not for $T \nu \mathrm{FDPF} 1$. We tested this hypothesis by measuring $\mathrm{H}_{2} \mathrm{O}_{2}$ produced by either $T \nu \mathrm{FDPF} 1$ or $T \nu \mathrm{FDPF} 3$ with Amplex Red in a discontinuous assay (Supplementary Table S3). We found that $T v$ FDPF1 produces $86 \pm 5 \% \mathrm{H}_{2} \mathrm{O}_{2}$ while $T v$ FDPF3 produces only $9.6 \pm 3.7 \%$.

\section{Purification of highly active holoTvFDPF3.}

We noticed that a minor peak but with extremely high specific activity eluted in the wash during the anion exchange purification step of $T v$ FDPF3. After modifying our purification scheme, we were able to significantly enrich and better separate this TvFDPF3 fraction, which had near-stoichiometric FAD and FMN occupancy $(0.94 \pm 0.09$ and $0.92 \pm 0.09)$ (Table 1, Fig. 3A). The apparent molecular mass of such preparations, which we refer to as "holoTvFDPF3", was $258 \pm 3 \mathrm{kDa}$, as judged by analytical gel-filtration (with a sharper appearance compared to both TvFDPF1 and 3). We used BN-PAGE as an independent method to assess the molecular weight of holoTvFDPF3 and observed an apparent molecular mass of 322 $\pm 24 \mathrm{kDa}$ (Supplementary Figure S8).

The absorption spectrum of holoTvFDPF3 had stronger features of flavin bands as well as of the rubredoxin-like center at 375, 451, 472 and $566 \mathrm{~nm}$ (Fig. 3B). Most importantly, the Michaelis-Menten analysis revealed that the $\mathrm{V}_{\max }$ of holoTvFDPF3 was $291 \pm 26 \mu \mathrm{mol} \mathrm{min}^{-1} \mathrm{mg}^{-1}$, a significant increase $(\sim 24$ times) compared to the low FMN occupancy $T \nu$ FDPF3. The $K_{\mathrm{MS}}$ for NADH and NADPH were $56 \pm 2 \mu \mathrm{M}$ and $427 \pm 160 \mu \mathrm{M}$, respectively and $k_{\text {cat }}$ (with NADH) was $466 \pm 42 \mathrm{~s}^{-1}$ per monomer (Table 2, Fig. 3C). The iron content of holoTvFDPF3 was $1.70 \pm 0.12$, which is 3 times higher compared to initially purified low activity $T v$ FDPF3 (Supplementary Table S2). We also used ICP-MS to characterize the metal content of holoTvFDPF3 and detected a small amount of $\mathrm{Zn}^{2+}$ that is likely incorporated in place of $\mathrm{Fe}^{2+/ 3+}$ in the diiron site (Supplementary Table S4). Other metal ions were present only in negligible quantities. Finally, the $\mathrm{H}_{2} \mathrm{O}_{2}$ byproduct formation was two-fold lower $(5.0 \pm 2.7 \%)$ than of initially purified $T v$ FDPF3 (Supplementary Table S3). In summary, holoTvFDPF3 had more complete FAD/FMN/Fe occupancy, as reflected in its increased specific activity and we employed it in all our subsequent experiments. 


\section{Titration of dithionite-reduced enzyme with $\mathrm{NAD}^{+}$.}

To directly demonstrate the entry point of reducing equivalents into holoTvFDPF3 we performed anaerobic titration with $\mathrm{NAD}^{+}$after initial reduction with dithionite (36). Oxidized $\mathrm{NAD}^{+}$addition causes the appearance of a long wavelength band centered at $704 \mathrm{~nm}$ (Fig. 3D). This broad band reflects stacking of $\mathrm{FADH}_{2}$ and $\mathrm{NAD}^{+}$planes (charge transfer complex) (36,37). This absorbance change is complete at exactly 1 equivalent of $\mathrm{NAD}^{+}$per enzyme monomer (each monomer of $T v$ FDPF3 contains one FAD and one FMN). This clearly suggests that although both flavins in the protein can be chemically reduced by dithionite only one flavin binds NAD ${ }^{+}$tightly. Since FMN of the Class A FDPs is not reduced by NADH, the observed behavior is consistent with the entry of NADH via $\mathrm{FADH}_{2}$ of the C-terminal NROR domain.

\section{Insights into redox centers of holoTvFDPF3 by EPR spectroscopy.}

The architecture of the fusion FDP system presents a unique opportunity to biophysically characterize redox centers that are amenable to Electron Paramagnetic Resonance (EPR) spectroscopy. FAD and FMN absorption in the visible region overwhelm the moderate absorption of the rubredoxin center while the diiron center does not absorb in the UV-Visible range. On the other hand, oxidized and reduced flavins are diamagnetic and thus invisible by EPR. In frozen samples at low temperature signals of semiquinone radicals can be detected but are saturated at the microwave power used and do not overlap. Thus, in the "as-isolated" holo $T \nu$ FDPF3 protein a very strong isotropic EPR signal at $g=4.3$ could easily be detected (Fig. 4A). This signal is typical for the oxidized Rb-like center, of which the $\mathrm{d}^{5}$ ferric ion is in a high spin state $(S=5 / 2)$. Such high spin ferric species exhibit three EPR signals from its Kramers' doublets, of which only the $\mid \pm 3 / 2>$ doublet shows an intense signal. Three almost identical $g$-values at 4.3 occur if the ratio of the spin Hamiltonian parameters $\mathrm{E}$ and $\mathrm{D}$ is above $0.3(|\mathrm{E} / \mathrm{D}|>0.3)$. Weak signals of the two other doublets (i.e. only the absorption-shaped signal corresponding to the highest $g$-value) could also be detected. The intensity of the temperature corrected $g=9.3$ EPR signal of the $\mid \pm 1 / 2>$ doublet decreased above $4 \mathrm{~K}$, indicating $\mathrm{D}=+(1.3 \pm 0.6) \mathrm{cm}^{-1}$ (Fig. 4B). In the $15 \mathrm{~K}$ minus $9 \mathrm{~K}$ difference EPR spectrum the $g=9.8$ EPR feature of the $\mid \pm 5 / 2>$ doublet could be revealed. For the determination of the redox midpoint potential at room temperature holoTvFDPF3 was oxidatively and reductively titrated in the presence of organic dyes by addition of potassium ferricyanide or sodium dithionite, respectively. When such samples were frozen in liquid nitrogen, the intensity of the $g=4.3$ EPR signal of the ferric state decreased upon reduction, in agreement with conversion to the EPR silent $S=2$ ferrous state of the Rb-like center (Fig. 4C). By fitting the intensity to the appropriate Nernst equation for a single electron process a midpoint potential of $-(56 \pm 10) \mathrm{mV}$ vs. $\mathrm{H}_{2} / \mathrm{H}^{+}$for the $\mathrm{Rb}$-like center was determined (Fig. 5A).

The EPR detection of the diiron center and therefore the analysis of its redox chemistry is inherently more difficult. First, the diferrous and diferric states are diamagnetic or have integer spin and are not (easily) detectable. Detection of the mixed-valence dinuclear center $\left(\mathrm{Fe}^{2+}-\mathrm{Fe}^{3+}\right)$ is hampered by the narrow temperature range at which the extremely anisotropic and large linewidth $S=1 / 2$ EPR signal can be detected. But at moderate microwave power at $7 \mathrm{~K}$ and by combining several preparations of holoTvFDPF3 we could detect a rhombic signal with $g=1.94,1.79$ and 1.53 in a sample titrated to a solution potential of $+115 \mathrm{mV}$ which was achieved by addition of menadiol (Fig. 4C). Menadiol (reduced menadione) was previously used to characterize the EPR spectrum of a Class F FDP from Clostridium difficile by Folgosa and colleagues (38). Due to the small quantity of the high activity holo enzyme and the weak EPR intensity it was not feasible to perform extensive redox titrations as in case of the Rb-like center. By combining three high activity preparations dye-mediated titrations with a total number of 7 datapoints enabled us to follow the EPR intensity of the mixed-valence EPR signal as a function of the solution potential. The diferric to mixed-valence potential was determined being $+(55 \pm 30) \mathrm{mV}$ (Fig. 5B). Line shape changes, possibly due to reduction of the nearby FMN or conformational changes, only allowed us to estimate the mixed valence to diferrous state midpoint potential to $-(180 \pm 50) \mathrm{mV}$ (Fig. 5B). 


\section{Redox titration of holoTvFDPF3 followed by the UV-Visible spectroscopy.}

To complete our characterization of the redox chemistry of holoTvFDPF3, visible spectroscopy at room temperature was employed to estimate the midpoint potentials of FAD and FMN in the presence of 5 $\mu \mathrm{M}$ mediator concentrations in a reductive titration. Flavin visible spectra of the protein bleached due to reduction to the hydroquinone states before onset of strongly absorbing viologen signals. At a wavelength of $425 \mathrm{~nm}$ the contribution of the mediators was relatively low and constant. After spectral subtraction the intensity of the sum of FMN and FAD visible contributions could be followed (Fig. 5C). In our experiments no flavin semiquinone signals were detected. Two separate redox potential ranges with $425 \mathrm{~nm}$ absorbance changes were observed, which based on the nearly stoichiometric presence of FMN and FAD likely correspond to redox transitions of the individual flavins. We assigned the lower flavin potential to the $\mathrm{FAD} / \mathrm{FADH}_{2}$ couple, as logical low potential entrance into the ET pathway. The best fit to the experimental data required equal potentials of $-235 \mathrm{mV}$ vs. $\mathrm{H}_{2} / \mathrm{H}^{+}\left(\mathrm{pH}\right.$ 7.5) for the two $\mathrm{n}=1$ transitions of $\mathrm{FADH}_{2}$ (Fig. 5C). A maximal population of $33 \%$ semiquinone can be calculated, which in its anionic form is apparently too weak to be detected among mediator and reduced FAD and FMN signals. FMN behaved almost like an $\mathrm{n}=2$ redox system with $-150 \mathrm{mV}$ vs. $\mathrm{H}_{2} / \mathrm{H}^{+}(\mathrm{pH} 7.5)$, i.e. extensive disproportionation of the semiquinone (max. $2 \%$ ) with estimated potentials of $-110\left(\mathrm{FMNH}_{2} / \mathrm{FMNH}\right)$ and $-190 \mathrm{mV}(\mathrm{FMNH} / \mathrm{FMN})$.

In summary, electrons within the multidomain system of $T v$ FDPF3 flow from NADH/NAD ${ }^{+}(-335$ $\mathrm{mV}$ at $\mathrm{pH} 7.5)$ to FAD $(-235 \mathrm{mV})$, rubredoxin $(-56 \mathrm{mV})$, FMN $(-150 \mathrm{mV})$ and ultimately on the dinuclear center $(-180$ and $+55 \mathrm{mV}$, average $-63 \mathrm{mV})$, the later one, in its reduced state reacts with dioxygen. We assume that the strong electrochemical driving force from $\mathrm{NADH}$ and $\mathrm{FADH}_{2}$ pushes the two times one electron transfer from the Rb-like center to the FMN within the FDP domain (see Discussion below).

\section{Molecular architecture of holoTvFDPF3.}

We used single-particle cryo-EM to investigate domain organization and redox center positioning in holo $T v$ FDPF3. Two-dimensional class-averages had clear secondary structure and were approximately 80-100 $\AA$ in diameter, consistent with the expected size of a globular monomer or a portion of a higher oligomer (Fig. 6A). Three-dimensional reconstruction of the particle set without symmetry enforced produced a $6.6 \AA$ resolution map (Supplementary Figs. S9, S10; Supplementary Table S5) with local resolutions ranging from $6.2 \AA$ at the core of the particle to $7.0 \AA$ at the periphery (Supplementary Fig. S10A). At this resolution, alpha helices could be clearly delineated but a model could not be constructed de novo. We modeled individual domains by threading the TvFDPF3 sequence onto structures of homologous proteins using PHYRE2 (39). The models were then manually docked into the cryo-EM density, revealing two FDP-like domains bound in a C2-symmetric dimer interface (Supplementary Fig. S11). Electron density was noticeably poorer for one subunit of the dimer, potentially as a result of conformational heterogeneity produced by hinge motions at the dimer interface. We refined the density map again with $\mathrm{C} 2$ symmetry applied, resulting in a symmetric map with 6.8 Å nominal resolution, clear density for both subunits of the dimer, and local resolutions ranging from $5.4 \AA$ to $6.9 \AA$ (Fig. 6B, Supplementary Fig. S12). Docking homology models of the FDP-like domain into the map with C2symmetry applied produced a model equivalent to that generated from the original map without symmetry applied. In the final C2-symmetric map, density was observed for the diiron and FMN moieties in the positions expected from previously reported structures of Class A FDPs (Supplementary Fig. S13).

The FDP-like domain of $T v$ FDPF3 forms an expected "head-to-tail" dimer that positions the diiron center of one protomer $8-10 \AA$ away from the FMN moiety bound in the neighboring subunit (Fig. 6C). However, the dimer interface differs from that previously reported in structures of dimeric Class A FDPs from D. gigas, G. intestinalis and M. thermoacetica $(14,17,19)$. The trans interaction between the large subdomain of one subunit and the small subdomain of its neighbor is similar to structures reported previously (all-atom RMSD 1.570), with a $12^{\circ}$ rotation about the redox center (Fig. 6D). The holoTvFDPF3 dimer interface differs from that observed for Class A FDP from $D$. gigas (ROO) as a result of a $70^{\circ}$ rotation about the linker connecting the large and small subdomains of the FDP-like core (Fig. 6E). This rotation substantially alters the interface between protomers (Supplementary Fig. S14). 


\section{Discussion}

There are substantial gaps in our understanding of core energy metabolism and redox maintenance in microaerophilic human parasites that belong to the Excavata supergroup $(4,40)$. Most of their proteomes, including key metabolic enzymes, are not well characterized and are annotated primarily based on sequence homology. In this work we aimed to close this knowledge gap by biochemical and structural studies of the oxygen detoxification systems reported in $T$. vaginalis (4). The natural function of $\mathrm{H}_{2} \mathrm{O}$-forming $\mathrm{NADH}$ oxidases (NOXes) and multicomponent flavodiiron proteins (FDPs) in microaerophilic human protozoan parasites is both regeneration of oxidized pyridine dinucleotides and constant $\mathrm{O}_{2}$ removal (detoxification) from the surroundings (Fig. 1A) $(3,4,41,42)$. In addition, these protozoa lack enzymes typically used to combat reactive oxygen species (ROS), including catalase and superoxide dismutase (SOD), and are therefore dependent on the ability of the $\mathrm{H}_{2} \mathrm{O}$-forming oxidase reaction to remove $\mathrm{O}_{2}$ before it can participate in the ROS-forming side reactions $(3,4,42)$. Better understanding of the $\mathrm{O}_{2}$ scavenging systems are needed as these pathways were recently suggested as targets for therapeutic interventions against various human protozoan parasites (3).

The presence of a NOX in T. vaginalis, and its general acceptance in the literature, was based on the original work of Linstead and Bradley from 1988 (3,4,9,31). In this study we show that in T. vaginalis the closet homologs of a typical NOX enzyme are three very similar in amino acid sequence Class F FDPs that are currently misannotated in databases (TVAG_263800, TVAG_049830 and TVAG_121610) (Fig. 2B, Supplementary Table S1). Based on domain organization all three proteins harbor N-terminal FDP, central Rb and C-terminal NROR domains (Fig. 2). Of note, Smutna and colleagues previously suggested that TVAG_263800, TVAG_049830 and TVAG_121610 proteins are "self-sufficient" FDPs, however, this prediction was not tested, as only a "stand-alone" Class A FDP from T. vaginalis (TVAG_036010) was biochemically characterized at that time (18).

To directly test whether these genes encode "self-sufficient" FDPs, we overexpressed corresponding protein products in E. coli (Supplementary Figure S4A-F). The initial kinetic characterization of these recombinant proteins revealed that despite variable and substoichiometric loading with FMN and iron, all three enzymes are capable of using NADH or NADPH as substrates (Tables 1-2, Supplementary Table S2). The highest $\mathrm{V}_{\max }\left(12 \pm 1 \mu \mathrm{mol} \mathrm{min} \mathrm{mg}^{-1}\right)$ was observed for $T v$ FDPF3 with NADH. Notably, the FMN content is negatively correlated with the off-target $\mathrm{H}_{2} \mathrm{O}_{2}$ production as $T v F D P F 1$ (only $1 \%$ of FMN per monomer) mostly produces $\mathrm{H}_{2} \mathrm{O}_{2}(86 \pm 5 \%$ ) (Supplementary Table S3). Once the FMN loading is $\sim 10 \%$ as in $T v F D P F 3$, the $\mathrm{H}_{2} \mathrm{O}_{2}$ production is significantly lower $(9.6 \pm 3.7 \%)$ and the $\mathrm{NADH}: \mathrm{O}_{2}$ stoichiometry is 2:1. Because the FMN cofactor is a part of the FeFe-FMN diiron active site, very low levels of FMN cofactor in $T v$ FDPF1 allow only a minor fraction of the four-electron $\mathrm{H}_{2} \mathrm{O}$-forming reaction to go until completion and the most of reducing equivalents are used within the C-terminal NROR domain to produce exclusively $\mathrm{H}_{2} \mathrm{O}_{2}$. A similar phenomenon has been reported for NROR from Clostridium acetobutylicum, which is a $\mathrm{H}_{2} \mathrm{O}_{2}$-forming $\mathrm{NADH}$ oxidase. When $C$. acetobutylicum $\mathrm{NROR}$ is mixed with fprA2 (Class A FDP) and $\mathrm{Rb}$, the NROR-Rb-fprA2 system starts to catalyze an efficient $\mathrm{H}_{2} \mathrm{O}$-forming oxidase reaction (23).

Next, we significantly optimized our purification scheme to obtain a highly active TvFDPF3 with near-stoichiometric amounts of both FAD and FMN cofactors (holoTvFDPF3) (Table 1, Fig. 3A-D). The iron content of holo $T \nu$ FDPF3 also improved to $1.7 \pm 0.12$ of Fe per monomer (Supplementary Table S2).

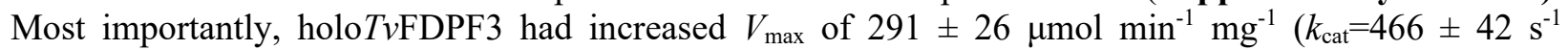
calculated per dimer) with $K_{\mathrm{M}}$ for NADH at $56 \pm 2 \mu \mathrm{M}$ (Table 2). We note that if an average of $1.3 \mathrm{Fe}$ is missing from each dinuclear center, the turnover number under $V_{\max }$ conditions could be as high as 1310 $\mathrm{s}^{-1}$, or as high as $820 \mathrm{~s}^{-1}$, if the missing $1.3 \mathrm{Fe}$ is equally distributed over both dinuclear and the rubredoxin sites ("all-or-none"). The activity we are reporting of holoTvFDPF3 is similar to that reported for Class A FDP from E. histolytica $\left(400 \pm 30 \mathrm{~s}^{-1}\right)$ (Supplementary Table S6). In a recent study, Class F FDP from Clostridium difficile was cloned and purified with iron and flavin content comparable to holoTvFDPF3 and a 30-fold lower of $k_{\text {cat }}$ of $16.0 \mathrm{~s}^{-1}$ was reported (Supplementary Table S6) (38).

Remarkably, the $V_{\max }$ we determined for holoTvFDPF3 $\left(466 \pm 42 \mathrm{~s}^{-1}\right)$ agrees well with the $V_{\max }$ number reported for the native $\mathrm{H}_{2} \mathrm{O}$-forming $\mathrm{NADH}$ oxidase preparation from $T$. vaginalis from the study 
by Linstead and Bradley $\left(470 \mathrm{~s}^{-1}\right)(31)$. In the original study authors presented the purification of an $\mathrm{H}_{2} \mathrm{O}-$ forming NADH oxidase activity that migrated as: (i) two close bands of $\sim 97-97.5 \mathrm{kDa}$ on the SDS-PAGE, and (ii) two very similar bands during isoelectric focusing (pIs 5.5) (31). This clearly represents a contradiction, as all known tDBDF family NOXes have molecular mass of $\sim 50 \mathrm{kDa}$. When we compared the pIs and molecular masses of $T \nu \mathrm{FDPF} 1-3$ they matched exactly the behavior of protein samples described by Linstead and Bradley (31). For example, the $\mathrm{pI}$ of $T \nu$ FDPF1 (5.25) and pIs of $T v$ FDPF2-3 (5.75 and 5.63) can explain the two very close bands during isoelectric focusing. During late stages of our study we learned that $T \nu$ FDPF2 was detected based on the mass spectrometric analysis in the cell lysate of $T$. vaginalis and resulted protein was in-gel stained for the NADH oxidase activity (neither specific activity nor $K_{\mathrm{M}}$ was reported) (43). In the same study, a purification of recombinant $T \nu$ FDPF2 for possible kinetic and spectroscopic analysis was not successful and neither $T \nu$ FDPF1 nor $T \nu$ FDPF3 were detected in cell lysates (43).

Our work complements a recent study by Folgosa and colleagues where authors determined redox potentials of redox cofactors that constitute the ET pathway within $C$. difficile FDPF (38). Here, we report a complete set of redox potentials that compose all four redox centers of the multi-domain $T v$ FDPF3 (Fig. 5, Supplementary Table S7). The redox potential of the FAD within the NROR domain (FAD, $\mathrm{E}_{\mathrm{m}}=-235$ $(\mathrm{n}=1)$ and $-235 \mathrm{mV}(\mathrm{n}=1))$ is very similar to potentials reported for $C$. difficile FDPF and "stand-alone" NRORs from E. coli and P. furiosus (Supplementary Table S7). The redox potential of the Fe(SCys) 4 center $\left(E_{m}=-56 \mathrm{mV}, \mathrm{n}=1\right)$ is very close to values reported in the literature for several "stand-alone" rubredoxins or the Rb-like domain of $C$. difficile FDPF (Supplementary Table S7). These values are very different from known redox potentials of the rubredoxin-like center of nigrerythrin and rubrerythrin $(+213$ to $+281 \mathrm{mV}$ ) which is surprising as the middle domain of FDPFs is maximally homologous to rubrerythrins. The redox potential of the FMN cofactor $\left(F M N, E_{m}=-190(n=1)\right.$ and $\left.-110 \mathrm{mV}(n=1)\right)$ is very similar to values reported for multiple Class A FDPs (Supplementary Table S7). Finally, in this study we were able to determine the reduction potential of the diiron center $\left(\mathrm{E}_{\mathrm{m}}=-180(\mathrm{n}=1)\right.$ and $\left.+55 \mathrm{mV}(\mathrm{n}=1)\right)$. We note that this value is different when compared to $G$. intestinalis FDPA $\left(\mathrm{E}_{\mathrm{m}}=+2\right.$ and $\left.+163 \mathrm{mV}\right)$ (Supplementary Table S7).

Our cryo-EM analysis revealed an unexpected architecture for the dimer interface of the N-terminal FDP core of holoTvFDPF3. In contrast to previous structures of the head-to-tail "stand-alone" Class A FDPs $(14,17,19)$, the subdomains of the FDP core exhibit a large rotation about the connecting linker, altering the dimerization interface while preserving the catalytic interface between the diiron center and FMN. We were unable to resolve electron density for Rb or NROR domains, suggesting that they may move flexibly relative to the central FDP-like core. This is not altogether unexpected, as the N-terminal FDP part is tethered to the other redox centers via an extended linker. It has been previously shown that Class A FDPs catalyze robust $\mathrm{H}_{2} \mathrm{O}$ formation when $\mathrm{Rb}$ and NROR are supplied in trans $(12,19,20,35)$. The tethering of domains within $T v F D P F 3$ may increase the effective concentration of these domains and facilitate short-lived electron-shuttling intermediate conformations that were not well populated in our micrographs. Moreover, the larger-than-expected molecular masses observed in both size-exclusion chromatography and native PAGE for TvFDPF1-3 suggest that the Rb-NROR domains contribute to an elongated protein shape (44). The reorganization of subdomains within the N-terminal FDP portion of $T \nu$ FDPF3 may hint at the interaction mechanism with the linked $\mathrm{Rb}$ and NROR domains. The subdomain rotation we observe in $T \nu \mathrm{FDPF} 3$ exposes a hydrophobic surface that in structures of similar proteins is occluded within the dimerization interface (Fig. 6F). This surface is positioned approximately $35 \AA$ away from the C-terminus of the nearest FDP-like small subdomain and approximately $10 \AA$ away from the diiron:FMN reaction center. Although we did not observe electron density in this portion of the map, the long linker connecting the FDP-like and $\mathrm{Rb}$ domains could bridge this distance, permitting this surface to bind the rubredoxin domain and complete electron transfer with the NROR domain.

Both our structural insight and biochemical characterization bring up an important question about the ET pathway within the dimer of holo $T \nu$ FDPF3. Here, we propose that the most parsimonious flow of reducing equivalents is as follows: $\mathrm{FAD} \rightarrow \mathrm{Fe}(\mathrm{SCys})_{4} \rightarrow \mathrm{FMN}$ within the subunit 1 and then the diiron center of the subunit 2 (Fig. 7). Spatial constraints make ET from the FAD to the $\mathrm{Fe}(\mathrm{SCys})_{4}$ center of the other 
subunit very unlikely. The dimerization interface holoTvFDPF3 lies with the N-terminal FDP core and therefore it is unlikely that $\mathrm{Rb}$ and NROR from different protomers exchange electrons just like Fe-Fe and FMN centers do (Fig. 7). In summary, the two active sites within the dimer of "head-to-tail" FDP are formed and electrons are relayed through Rb-NROR paths separately in both subunits 1 and 2 (Fig. 7). Testing our hypothesis of electron transfer from NROR to the FMN and potential asymmetry in the ET pathway within active sites of the dimer of $T \nu \mathrm{FDPF} 3$ is the next phase of our study.

\section{Experimental procedures}

\section{Materials}

All chemicals were purchased from Sigma-Aldrich, VWR and Fisher Scientific unless otherwise specified. Menadiol (2-methyl-1,4-naphthalenediol) was from AK Scientific (Union City, CA).

\section{Bioinformatic Analysis}

The EuPathDB Bioinformatics Resource Center (https://eupathdb.org/eupathdb/) was used to browse genomic sequences of $T$. vaginalis $\mathrm{C} 3$ and other eukaryotic microbes (45). Multiple sequence alignments were constructed using a stand-alone version of ClustalX 2.0 and edited using BOXSHADE 3.21 .

\section{Cloning of TvFDPF1-3 with a C-terminal His6tag}

Genomic DNA of Trichomonas vaginalis obtained from ATCC (Strain C-1:NIH [ATCC 30001]) was used to PCR amplify the gene sequences encoding $T v$ FDPF1-3. The following primers were used to clone TVAG_263800 (TvFDPF1) contained NdeI and XhoI restriction sites (underlined): forward 5' - TTA ATT CAT ATG CTT AAA ATT CAG CAA CTT ACA GAA GAT ATC - 3' and reverse 5' - TTA ATT CTC GAG AAA CAA TTC AGC AAG AAC GTG CTG TAA AGT ATG - 3'. The following primers were used to clone TVAG_049830 (TvFDPF2) contained NdeI and KpnI restriction sites (underlined): forward 5' - TTA ATT CAT ATG CTT AAA ATA CAG CAG CTC ACT GAA GAC - 3' and reverse 5' - TTA ATT GGT ACC TT GAA GAT TTC AGC CAT GAT GCG CTC TAA TG - 3'. The following primers were used to clone TVAG_121610 (TvFDPF3) contained AseI (creates an overhang compatible with the NdeI restriction site) and XhoI restriction sites (underlined): forward 5'- TTA ATT ATT AAT ATG CTT AAA ATT CAG CAG CTT ACA GAA GAT - 3' and reverse 5' - TTA ATT CTC GAG AAA TAC CTC AGC AAC AAC ACG CTG CAT TGA GTG - 3'. All resulting PCR products were cloned into the pET30a expression vector (Novagen, EMD Millipore).

\section{Initial expression and purification of TvFDPF1-3}

All proteins in this study were overproduced in One Shot BL21 Star (DE3) chemically competent Escherichia coli (ThermoFisher [formerly Invitrogen], C601003). Bacterial culture was grown at $37^{\circ} \mathrm{C}$ in six $2.8 \mathrm{~L}$ flasks, each containing $1 \mathrm{~L}$ of Luria-Bertani medium (Becton, Dickinson and Company, 244610) supplemented with $50 \mu \mathrm{g} / \mathrm{ml}$ kanamycin until the absorbance at $600 \mathrm{~nm}$ reached $0.4-0.6$. At that point, the temperature was decreased to $15^{\circ} \mathrm{C}$ and bacterial culture was grown for additional 2 hours. After supplementation with $400 \mu \mathrm{M}$ ammonium iron(II) sulfate hexahydrate and $0.1 \mathrm{mM}$ isopropyl $\beta$-D-1thiogalactopyranoside bacterial cultures were grown overnight. For protein purification $E$. coli cell pellet was resuspended in $120 \mathrm{~mL}$ of $50 \mathrm{mM}$ sodium phosphate, $\mathrm{pH} 8.0,500 \mathrm{mM} \mathrm{NaCl}, 30 \mathrm{mM}$ imidazole (buffer A) containing $1 \mathrm{mg} / \mathrm{mL}$ of lysozyme, four cOmplete, EDTA-free, protease inhibitor cocktail tablets (Roche), $60 \mu \mathrm{L}$ benzonase nuclease (EMD Millipore) and $4 \mathrm{mM}$ phenylmethylsulfonyl fluoride (PMSF) and were disrupted by sonication on ice for $20 \mathrm{~min}$ at 30 -s intervals separated by 60 -s cooling periods. Following centrifugation, cell lysate was passed through a $0.4 \mu \mathrm{m}$ syringe filter, diluted to $\sim 300-400 \mathrm{~mL}$ with buffer A and loaded onto a $20 \mathrm{~mL}$ HisPrep FF 16/10 column (GE Healthcare/Cytiva). All chromatographic steps were performed using an AKTA Pure (GE Healthcare) or NGC Quest 10 Plus (Bio- 
Rad) chromatography systems. After washing HisPrep FF 16/10 column with $500 \mathrm{~mL}$ of buffer A, the gradient of 30-300 mM imidazole in buffer A was applied over $300 \mathrm{~mL}$. Fractions containing TvFDPF1-3 had distinct brown color and were exchanged into $50 \mathrm{mM}$ sodium phosphate, $\mathrm{pH} 7.5$ (buffer $\mathrm{B}$ ). In the next step, protein sample in $40 \mathrm{~mL}$ of buffer B was applied onto a $30 \mathrm{~mL}$ Source 15Q column equilibrated with $50 \mathrm{mM}$ sodium phosphate, $\mathrm{pH} 7.5,50 \mathrm{mM} \mathrm{NaCl}$ (buffer C) at a flow rate of $6 \mathrm{~mL} / \mathrm{min}$. Subsequently Source 15Q column was washed with $150 \mathrm{~mL}$ of buffer $\mathrm{C}$ and protein was eluted with $450 \mathrm{~mL}$ of gradient of 50$300 \mathrm{mM} \mathrm{NaCl}$ in buffer C. Size-exclusion chromatography was performed on a HiLoad 16/600 Superdex 200 (GE Healthcare/Cytiva, 28989335) or HiPrep 16/60 Sephacryl S-400 HR (GE Healthcare/Cytiva) columns equilibrated with $50 \mathrm{mM}$ sodium phosphate, $\mathrm{pH} 7.5,150 \mathrm{mM} \mathrm{NaCl}$ (buffer D) or with $50 \mathrm{mM}$ HEPES-NaOH pH 7.5, $150 \mathrm{mM} \mathrm{NaCl}$ buffer (buffer E). Fractions containing protein of interest were pooled, concentrated and flash-frozen in liquid nitrogen. Only affinity purification and size-exclusion chromatography steps were used for $T v \mathrm{FDPF} 2$ due to its low stability and tendency to precipitate.

\section{Determination of molecular weight by gel-filtration}

Analytical gel-filtration was performed using a Superdex 200 Increase 10/300 GL column (GE Healthcare/Cytiva) operated at $0.9 \mathrm{~mL} / \mathrm{min}$ in buffer E. Calibration curve was produced using aldolase (158 $\mathrm{kDa})$, conalbumin $(75 \mathrm{kDa})$ and ovalbumin $(44 \mathrm{kDa})$ from the high molecular weight gel-filtration calibration kit (GE Healthcare/Cytiva).

\section{Blue-native polyacrylamide gel electrophoresis (BN-PAGE)}

All BN-PAGE reagents were from ThermoFisher Scientific. The anode buffer was prepared using $30 \mathrm{~mL}$ of 20x NativePAGE running buffer and $570 \mathrm{~mL}$ of milliQ water. Two hundred $\mathrm{mL}$ of "the light" and "the dark" cathode buffers were prepared using milliQ water, $10 \mathrm{~mL}$ of $2 X$ NativePAGE running buffer and $1 \mathrm{~mL}$ or $10 \mathrm{~mL}$ of cathode buffer additive. Protein samples were prepared using $4 \mathrm{x}$ NativePAGE sample buffer. Before the electrophoresis wells of a 3-12 \% Novex Bis-Tris precast gel were filled with "the dark" cathode buffer. Samples and NativeMARK protein standards were added to assembled electrophoresis cell before "the dark" buffer was used to completely cover the inner chamber. Initial step of the electrophoresis was performed at $150 \mathrm{~V}$ until the dye front reached approximately one third of the gel. Then "the dark" cathode buffer in the inner chamber was replaced with "the light" cathode buffer and the run was continued till completion at $250 \mathrm{~V}$. We also used recombinant $\mathrm{H}_{2} \mathrm{O}$-forming NADH oxidases from L. brevis and $G$. intestinalis as additional molecular mass controls (11).

\section{Purification of highly active holoTvFDPF3}

The most active holoTvFDPF3 enzyme was obtained using the protocol described above but with several modifications. E. coli cells were grown in $6 \mathrm{~L}$ of Terrific Broth (TB) medium and for the homogenization step cell pellet was resuspended in $400 \mathrm{~mL}$ of buffer A supplemented with $1 \mathrm{mg} / \mathrm{mL}$ of lysozyme, six cOmplete, EDTA-free protease inhibitor cocktail tablets (Roche), $120 \mu \mathrm{L}$ benzonase nuclease and $4 \mathrm{mM}$ (PMSF). Affinity chromatography was performed using a self-packed $35 \mathrm{~mL}$ Ni Sepharose 6 Fast Flow column. For the anion-exchanger step the Source 15Q column was equilibrated with $50 \mathrm{mM}$ sodium phosphate, $\mathrm{pH} 7.5,15 \mathrm{mM} \mathrm{NaCl}$ (buffer F). The most active holoTvFDPF3 (fully loaded with both FAD and FMN and superior specific activity in the 160-210 U/mg range) was eluting as a distinct peak during the $150 \mathrm{~mL}$ wash step with buffer $\mathrm{F}$. We note that most of the protein was eluting in the gradient as described in the section above, but that protein fraction had very low specific activity compared to the highly active holoTvFDPF3. Size-exclusion chromatography was performed in buffer E.

\section{UV-Visible Spectroscopy}

Cary 100 and Cary 3500 UV-Visible spectrophotometers (Agilent) were used to record UV-visible spectra and perform activity assays under aerobic conditions. Anaerobic experiments were performed using Shimadzu 1900 UV-Visible spectrometer installed inside a glove box (Coy Laboratory Products). Concentration of free FAD and FMN in solution were determined spectrophotometrically using extinction coefficients for FAD at $450 \mathrm{~nm}\left(11.3 \mathrm{mM}^{-1} \mathrm{~cm}^{-1}\right)$ and for FMN at $446 \mathrm{~nm}\left(12.2 \mathrm{mM}^{-1} \mathrm{~cm}^{-1}\right)$ (46). 


\section{Enzymatic Assays}

Enzyme activity was monitored by following the decrease of the absorbance of NADH or NADPH at $340 \mathrm{~nm}$. A typical reaction mixture in $0.2 \mathrm{~mL}$ of the buffer $\mathrm{E}$ was incubated for $3 \mathrm{~min}$ at $37^{\circ} \mathrm{C}$ before $\mathrm{NAD}(\mathrm{P}) \mathrm{H}(0.5-600 \mu \mathrm{M})$ and enzyme $(0.1-10 \mu \mathrm{g})$ were added. An extinction coefficient $\left(\varepsilon_{340}=6.2 \mathrm{mM}^{-}\right.$ ${ }^{1} \mathrm{~cm}^{-1}$ ) was used to calculate NAD(P)H oxidase activity. The $k_{\text {cat }}$ values for $T v$ FDPF1-3 were calculated per dimer of the protein.

Near-simultaneous oxygen and NADH consumption were monitored using a custom-made instrument for measuring fluorescence spectra and time-resolved phosphorescence originally engineered for bioenergetics experiments with a suspension of purified mitochondria (the Mootha laboratory, Massachusetts General Hospital, Boston, MA). In the current set-up NADH was monitored by its autofluorescence $\left(\lambda_{\mathrm{ex}}=365 \mathrm{~nm} ; \lambda_{\mathrm{em}}=440-460 \mathrm{~nm}\right)$ and oxygen was measured using the oxygen phosphorescence sensor spot SP-PSt6-NAU (PreSens Precision Sensing GmbH, Regensburg, Germany) affixed inside a quartz cuvette. Generally, enzyme $(5-60 \mu \mathrm{g})$ and $\mathrm{NAD}(\mathrm{P}) \mathrm{H}(100-500 \mu \mathrm{M})$ were added to $0.5 \mathrm{~mL}$ of the buffer $\mathrm{E}$ at $28{ }^{\circ} \mathrm{C}$ under regular oxygen tension. The instrument was operated using homemade software developed in LabView, Matlab and Arduino Software IDE. Data from each experiment was exported as a text file and analyzed using SigmaPlot 13.0.

\section{Determination of FAD and FMN content}

Protein samples $(10-50 \mu \mathrm{M}$ of monomers) or FAD/FMN standards $(0-100 \mu \mathrm{M})$ in the buffer E or ultrapure water were incubated at $95{ }^{\circ} \mathrm{C}$ for $10 \mathrm{~min}$. After centrifugation at maximum speed a $50-\mu \mathrm{L}$ aliquot was added to $200 \mu \mathrm{L}$ of $50 \% / 50 \%$ methanol/acetonitrile solution and the resulting samples were subjected to the LC-MS analysis. For the LC-MS analysis, running buffer $\mathrm{G}$ was $5 \%$ acetonitrile, $20 \mathrm{mM}$ ammonium acetate and $0.25 \%$ ammonium hydroxide, $\mathrm{pH} 9.0$ while running buffer $\mathrm{H}$ was $100 \%$ acetonitrile. The gradient was run on a Dionex Ultimate 3000 system with an Xbridge amide column $(2.1 \mathrm{x} 100 \mathrm{~mm}, 2.5 \mu \mathrm{m}$ particle size) at $220 \mu \mathrm{L} / \mathrm{min}$ flow rate, and started at $85 \% \mathrm{H}$ for 0.5 minutes, ramped to $35 \% \mathrm{H}$ over the next 3.5 minutes, ramped to $2 \% \mathrm{H}$ over the next 2 minutes, held at $2 \% \mathrm{H}$ for 1 minute, ramped to $85 \% \mathrm{H}$ for 1.5 minutes, then held at $85 \% \mathrm{H}$ for 1.5 minutes (and ramping to $420 \mu \mathrm{L} / \mathrm{min}$ ). The total run time was 12 minutes. The MS analysis was performed on a Thermo QExactive Orbitrap mass spectrometer operated in polarity switching mode with a scan range of $70-1000 \mathrm{~m} / \mathrm{z}$ and a resolving power of 70,000 at $200 \mathrm{~m} / \mathrm{z}$. The resulting data for FAD and FMN were processed using Thermo Xcalibur software.

The FAD/FMN content was also analyzed on an Agilent 6495 QqQ with jet stream source coupled to an Agilent 1290 LC stack with an Agilent HILIC-Z $(2.1 \times 150 \mathrm{~mm})$ column at the Scripps Center for Metabolomics (Department of Chemistry, TSRI). The mobile phase was composed of buffer I $=10 \mathrm{mM}$ ammonium acetate, $5 \mu \mathrm{M}$ medronic acid, $\mathrm{pH}=9$ and buffer $\mathrm{K}=90: 10$ acetonitrile/water, $10 \mathrm{mM}$ ammonium acetate, $5 \mu \mathrm{M}$ medronic acid, $\mathrm{pH}=9$. The gradient started at $98 \% \mathrm{~K}(0-1 \mathrm{~min})$ decreasing to $40 \% \mathrm{~K}(1-5 \mathrm{~min})$ and was followed by an isocratic step ( $5 \mathrm{~min}-7 \mathrm{~min}$ ) before a $5 \mathrm{~min}$ post-run for column re-equilibration. The flowrate was set to $250 \mu \mathrm{L} / \mathrm{min}$ and the sample injection volume was $5 \mu \mathrm{L}$. Operating in negative ion mode, the source conditions were as follows: drying gas temperature set to $200{ }^{\circ} \mathrm{C}$ with a flowrate of $11 \mathrm{~L} / \mathrm{min}$, the sheath gas temp was $300{ }^{\circ} \mathrm{C}$ with a sheath gas flowrate of $12 \mathrm{~L} / \mathrm{min}$, the neb pressure set to $35 \mathrm{psi}$, cap voltage set to $2500 \mathrm{~V}$ and nozzle voltage set to $1500 \mathrm{~V}$. Data was processed using Agilent MassHunter Quantitative analysis software. All samples had values that were within the range of the calibration standards.

\section{Iron determination with Ferene}

Proteins were diluted in buffer E to $10-40 \mu \mathrm{M}$ of monomers. Iron standards were prepared using Mohr's Salt (ammonium iron(II) sulfate hexahydrate) in ultrapure water. Subsequently $100 \mu \mathrm{L}$ of $1 \%$ hydrochloric acid were added to $100-\mu \mathrm{L}$ aliquots of protein samples or iron standards and tubes were incubated at $80{ }^{\circ} \mathrm{C}$ for $10 \mathrm{~min}$. Next, $500 \mu \mathrm{L}$ of $7.5 \%$ ammonium acetate were added with subsequent addition of $100 \mu \mathrm{L}$ of $4 \%$ ascorbic acid, $100 \mu \mathrm{L}$ of $2.5 \%$ sodium dodecylsulphate and $100 \mu \mathrm{L}$ of iron chelator 3-(2-pyridyl)-5,6-bis(5-sulfo-2-furyl)-1,2,4-triazine disodium salt (samples were vortexed after each 
addition). After centrifugation at $13000 \mathrm{xg}, 800 \mu \mathrm{L}$ of each sample were transferred to $1 \mathrm{~mL}$ plastic cuvettes to record absorbance at $592 \mathrm{~nm}$.

\section{Determination of $\mathrm{H}_{2} \mathrm{O}_{2}$ production}

$\mathrm{H}_{2} \mathrm{O}_{2}$ production was monitored in a discontinuous assay. Large excess of the protein $(5-30 \mu \mathrm{g})$ was added to the assay mixture that contained $110 \mu \mathrm{M} \mathrm{NADH}$ in $0.3 \mathrm{~mL}$ of buffer $\mathrm{E}$ and allowed to run at room temperature for $5 \mathrm{~min}$ (to establish full conversion of NADH to $\mathrm{H}_{2} \mathrm{O}_{2} / \mathrm{H}_{2} \mathrm{O}$ ). Aliquots of $50 \mu \mathrm{L}$ were taken and added to another $50 \mu \mathrm{L}$ of Buffer E supplemented with $2 \mu \mathrm{L}$ of HRP (Abcam, ab102500), and 2 $\mu \mathrm{L}$ Amplex Red (Abcam, ab102500). In parallel a calibration curve with known amounts of $\mathrm{H}_{2} \mathrm{O}_{2}$ standards was constructed. Ten minutes later after incubating the assay mixture in a clear 96-well plate, the absorbance at $600 \mathrm{~nm}$ was recorded using EnVision 2103 plate reader.

\section{Metal content determination by ICP-MS}

The ICP-MS analysis was done on a Thermo Scientific iCAP RQ ICP-MS in the Environmental and Complex Analysis Laboratory (UCSD). Protein samples were prepared at $0.5 \mu \mathrm{M}$ of active sites (10 $\mathrm{mL}$ ) in $2 \%$ Trace Metal Nitric Acid and analyzed directly. The analysis was conducted in Kinetic Energy Discrimination (KED) mode monitoring 45Sc and $89 \mathrm{Y}$ as internal standards.

\section{EPR spectroscopy}

The midpoint potentials of the rubredoxin and dinuclear iron centers of holoTvFDPF3 were determined from EPR signal intensities of the oxidized (as purified) and mixed-valence state, respectively. About $5 \mathrm{mg} / \mathrm{mL}$ holoTvFDPF3 in (end volume $2 \mathrm{~mL}$ for the reductive titration with sodium dithionite and $1.5 \mathrm{~mL}$ for the oxidative titration with potassium ferricyanide) $50 \mathrm{mM}$ HEPES-NaOH, pH 7.5, were stirred under anaerobic conditions at $298 \mathrm{~K}$. The solution potential was measured with an InLab ARGENTHAL (Mettler, Germany) microelectrode $\left(\mathrm{Ag} / \mathrm{AgCl},+207 \mathrm{mV}\right.$ vs. $\mathrm{H}_{2} / \mathrm{H}^{+}$with in-built $\mathrm{Pt}$ counter electrode) in the presence of phenazine ethosulfate, methylene blue, resorufin, indigo carmine, 2-hydroxy-1,4naphthoquinone, $\quad N, N, N, N$-tetramethyl-p-phenylendiamine, sodium anthraquinone-2-sulfonate, phenosafranin, safranin T, neutral red, benzyl- and methylviologen (all at final concentration of $20 \mu \mathrm{M}$ ). After adjustment of the potential by microliter additions of the sodium dithionite or potassium ferricyanide and 5 min equilibration, samples were withdrawn, removed from the anaerobic glove box in EPR tubes closed with ID $3 \mathrm{~mm}$ x OD $7 \mathrm{~mm}$ natural rubber tubing with $5 \mathrm{~mm}$ OD acrylic glass round stick. Samples were stored in liquid nitrogen until EPR spectra were recorded. For the menadiol-treated sample $300 \mu \mathrm{L}$ of $7 \mathrm{mg} / \mathrm{mL}$ holoT $\mathrm{FDP} 3$ with the first five mediators (at $10 \mu \mathrm{M}$ ) were titrated to a potential of $+115 \mathrm{mV}$ vs. $\mathrm{H}_{2} / \mathrm{H}^{+}$with sodium dithionite. Thereafter menadiol was added to a final concentration of $50 \mu \mathrm{M}$. EPR spectra were recorded with a digitally upgraded Bruker Elexsys E580 X band spectrometer with a 4122HQE cavity, an Oxford Instruments ESR 900 helium flow cryostat and the cryocooling system composed of a Stinger (Cold Edge Technologies) closed-cycle cryostat linked to an F-70 Sumitomo helium compressor. EPR tubes were produced by a local glassblower from Ilmasil PN tubing with OD $4.7 \mathrm{~mm}$ and $0.5 \mathrm{~mm}$ wall thickness obtained from Qsil (Langewiesen, Germany).

\section{$U V$-visible redox titrations}

$T v$ FDPF3 ( $1 \mathrm{mg}$ in $1 \mathrm{~mL}$ of $50 \mathrm{mM}$ HEPES-NaOH, $\mathrm{pH}$ 7.5) was completely reduced by addition of sodium dithionite ( $1 \mathrm{mM}$ final concentration). Increments of a $0.2 \mu \mathrm{M} \mathrm{NAD}{ }^{+}$in the same buffer were added. The UV-visible redox titration using a mixture of redox mediators (see section above) was performed by monitoring the absorbance at $425 \mathrm{~nm}$ using a Shimadzu 1900 UV-Visible spectrometer installed inside the Coy glove box.

\section{Half reaction of TVFDPF3 in presence of NONOate}

A stock solution $(10 \mathrm{mM})$ of diethylamine NONOate sodium salt hydrate (Sigma-Aldrich, Taufkirchen, Germany) was prepared by dissolving $3.9 \mathrm{mg}$ in $245 \mu \mathrm{L}$ of deoxygenated KOH (prepared by bubbling nitrogen in the anaerobic tent). Transfer of electrons from NADH to NO in the reaction catalysed 
by $T v$ FDPF3 was monitored at $340 \mathrm{~nm}$ using a Shimadzu UV-310 spectrophotometer in the Coy tent. To release NO the assay buffer, NADH $(200 \mu \mathrm{M})$ and NONOate $(100 \mu \mathrm{M})$ were mixed and incubated for 5 min. Next, $120 \mu \mathrm{g}$ of the enzyme were added, and absorbance or spectral changes were followed as a function of time. Samples where enzyme or NONOate were omitted served as controls.

\section{Cryo-EM sample preparation and data collection}

Purified highly active holoTvFDPF3 was diluted to $0.8 \mathrm{mg} / \mathrm{mL}$ using buffer $\mathrm{E}$ and $4 \mu \mathrm{L}$ was applied to a glow-discharged Quantifoil 200 mesh 1.2/1.3 Cu holey carbon grid (Electron Microscopy Sciences, Hatfield, PA). Excess sample was removed by blotting with a Vitrobot Mark IV (ThermoFisher) for $5 \mathrm{~s}$ at +15 blotting force in an environment held at $22{ }^{\circ} \mathrm{C}$ and $100 \%$ relative humidity, then the grid was frozen by plunging into liquid ethane. The frozen grid was imaged at $300 \mathrm{kV}$ with a Titan Krios microscope (ThermoFisher) with a slit energy filter (Gatan) set to $20 \mathrm{eV}$. Images were acquired in counting mode (105,000x nominal magnification, $0.825 \AA$ pixel size) on a K3 direct electron detector (Gatan). Acquisitions were stored as 51-frame dose-weighted movies with defocus ranging from -1.3 to $-2.5 \mu \mathrm{m}$. Each movie was collected with a $1.8 \mathrm{~s}$ total exposure time, a $30.3 \mathrm{e}^{-} \AA^{-2}$ frame $\mathrm{e}^{-1}$ specific dose, and a $54.5 \mathrm{e}^{-} \AA^{-2}$ overall electron dose. Serial-EM 3.8.6 was used to automate multi-shot image acquisition, and 7,398 total movies were collected (47).

\section{Cryo-EM data processing}

The holoTvFDPF3 image dataset was processed using CryoSPARC 3.2.0 to pick an initial particle set and RELION 3.1.3 to curate particles and refine maps (Supplemental Fig. S9) $(48,49)$. All micrographs were imported into CryoSPARC and preprocessed using the CryoSPARC internal Patch Motion Correction and Patch CTF Estimation jobs. An initial particle set was generated by blob picking particles between 80 $\AA$ and $180 \AA$ in diameter from all micrographs. These particles were twice sequentially subjected to $2 \mathrm{D}$ classification, and the best classes selected. The micrographs were then manually curated to remove images with minimum CTF fits of $9 \AA$ or greater. After curation, 6,938 of the original 7,398 movies remained under consideration. The $2 \mathrm{D}$ classes selected from blob picking were used to train a Topaz model, and the model was used to pick particles from the curated micrographs (50). Duplicate particles were removed by excluding particles with a center within $125 \AA$ of another particle. The coordinates of the remaining 1,511,632 particles were then encoded into star files for import into RELION using a custom python script, made available at https://github.com/tribell4310/reliosparc. The original set of 7,398 movies was separately imported into RELION and preprocessed using RELION's implementations of MotionCor2 1.2.6 and CTFFIND 4.1.13 (51,52). STAR files containing the coordinates of particles selected in cryoSPARC were then imported into RELION and used to extract particles from the RELION-preprocessed movies with a 256 pixel box size and no binning. The particle stack was subjected to three sequential rounds of 2D classification, with the best classes selected in each iteration. The particles were re-centered by 3D autorefinement using an initial model generated de novo in RELION from a larger set of curated particles. The re-centered particles were then re-extracted from micrographs with a 256 pixel box size and no binning using the refined particle coordinates. These particles were subjected to an additional cycle of 2D classification to remove any particles that would not align well after re-centering. The best classes were then 3D auto-refined, and the resulting volume was used to create a mask with a 9 pixel extension and a 7 pixel soft edge. The mask was then applied during 3D classification of the particle set into 6 classes using a tau_fudge parameter of 20, as has previously been used to resolve structural features in small targets (53). Two density types emerged from 3D classification: a compact map with well-resolved features and a lowresolution extended map with poor density, which may correspond to a more flexible conformation of the protein. Subsequent masked 3D classification of the compact classes did not improve the quality of the map, so all 101,628 particles in the compact conformation were included in the final particle stack. The compact conformation particles were auto-refined twice with progressively tighter masks, first with a mask with a 9 pixel extension and a 7 pixel soft edge, and then with a 7 pixel extension and a 7 pixel soft edge. The last masked refinement was then subjected to three sequential rounds of RELION's CTF refinement protocol, producing a map with $7.0 \AA$ A overall resolution. Finally, the map was sharpened by applying a B- 
factor of -534.7 , estimated during the RELION postprocessing protocol. The final sharpened map had an overall resolution of $6.6 \AA$. After modeling indicated likely C2 symmetry in the map, the set of 130,196 particles from 2D classes represented in the C1 map was three-dimensionally classified with $\mathrm{C} 2$ symmetry applied, a $150 \AA$ diameter spherical mask, a tau fudge factor of 20, and the C1 refined map lowpass filtered to $50 \AA$ as an input template. Two similar compact classes emerged and were subjected to a subsequent round of unmasked 3D classification. A homogeneous class containing 53,709 particles was identified and $3 \mathrm{D}$ auto-refined with a mask ( 6 pixel extension, 4 pixel soft edge), then CTF refined using the same protocol as above, resulting in a $6.6 \AA$ unsharpened map. The map was sharpened with a B-factor of -658.7 using the RELION postprocessing protocol, producing a final map with $6.8 \AA$ nominal resolution. Local resolutions were estimated using RELION's integrated local resolution estimation algorithm (54).

\section{Model Building}

Because the resolution of the final maps was insufficient to build atomic models de novo, the model was constructed using homology models from individual domains. Homology models were generated using the PHYRE2 server, and with $92 \%$ of the TvFDPF3 sequence covered between three models (39). Residues 2-366 (N-terminal FDP-like domain) were modeled onto the PDB 1VME structure (Class A FDP from Thermotoga maritima), residues 428-472 (rubredoxin domain) were modeled onto the PDB 1LKO structure (rubrerythrin from Desulfovibrio vulgaris), and residues 482-871 (the C-terminal NADH:rubredoxin oxidoreductase domain) were modeled onto the PDB 3NTA structure (NADH-dependent persulfide reductase from Shewanella loihica). Appropriate ligands were included in the homology models based on their conserved binding pockets in homologous structures. The map was consistent with a dimer of FDPlike domains, and models were built by manually placing homology models in density using UCSF Chimera (55), with the N- and C-terminal subdomains of the FDP-like domain separated to facilitate the large rotation between the subdomains. Once the subdomains were confidently placed, we verified that the domain placements were topologically reasonable by modeling the connecting linker region using the nextgeneration kinematic closure loop modeling protocol in Rosetta (56). The model was refined to the C2symmetric map using a single application of real-space refinement in Phenix (57). Structural alignments comparing $T \nu$ FDPF3 with the head-to-tail dimer form of rubredoxin:oxygen oxidoreductase from $D$. gigas (PDB 1E5D) were performed using PyMol (58). Figures were generated using UCSF Chimera and PyMol $(55,58)$.

\section{Data availability}

The Cryo-EM maps of TvFDPF3 have been deposited into the EMDB (accession \#: EMD-25790, EMD25787), and the underlying particle images have been deposited with EMPIAR (accession \#: EMPIAR10895). The docked homology models have been deposited in Zenodo (DOI: 10.5281/zenodo.5795907). All the other data are contained within this manuscript.

\section{Supporting information}

This article contains supporting information.

\section{Acknowledgements}

Cryo-EM data were collected at the Harvard Cryo-EM Center for Structural Biology at Harvard Medical School. We thank Xue Fei (Massachusetts Institute of Technology) and Michelle Fry (MGH) for helpful feedback during cryo-EM map refinement.

\section{Author contributions}

V.C. and V.K.M. conceived the study. V.C. cloned initial DNA constructs. E.N.A. with the help from V.C. and M.L.H. performed protein purification and protein characterization experiments. B.R. and A.J.P. designed and performed EPR and redox titrations experiments. B.R. performed activity assays with nitric oxide. T.A.B. and L.H.C. determined the cryo-EM structure. O.S.S. contributed to the mass spectrometry 
analysis. M.A.Y. assisted in oxygen consumption measurements. V.C. and E.N.A. analyzed the data and wrote the manuscript with the exception of the EPR spectroscopy section (co-written by B.R., A.J.P.) and the structural biology section (co-written by T.A.B., L.H.C.). All authors contributed to editing the manuscript and approved the final version. V.K.M. supervised initial experiments of this study. V.C. supervised all aspects of the study.

\section{Funding and additional information}

This work was supported by grants from the National Institutes of Health (R00GM121856 and R35GM142495 to V.C., R35GM142553 to L.H.C., F32GM133047 to O.S.S., R35GM122455 and TR01GM099683 to V.K.M., R00AG042026 and R01AA027097 to M.A.Y.) and the Helen Hay Whitney Foundation (to T.A.B.). V.K.M. is an Investigator of the Howard Hughes Medical Institute. A.J.P. acknowledges the DFG and the government of Rhineland-Palatinate for the upgrade of the EPR spectrometer.

\section{Conflict of interests}

V.K.M. and V.C. are listed as inventors on a patent application filed by Massachusetts General Hospital on the therapeutic uses of water forming NADH oxidases. V.K.M. is a scientific advisor to and receives equity from 5AM Ventures and Janssen Pharmaceuticals. O.S.S. was a paid consultant for Proteinaceous Inc.

\section{References}

1. Hodges, A. L., and Holland, A. C. (2018) Common Sexually Transmitted Infections in Women. Nurs Clin North Am 53, 189-202

2. Mark, H., Dhir, A., and Roth, C. (2015) CE: Sexually Transmitted Infections in the United States: Overview and Update. Am J Nurs 115, 34-44, quiz 45-36

3. Leitsch, D., Williams, C. F., and Hrdy, I. (2018) Redox Pathways as Drug Targets in Microaerophilic Parasites. Trends Parasitol 34, 576-589

4. Muller, M., Mentel, M., van Hellemond, J. J., Henze, K., Woehle, C., Gould, S. B., Yu, R. Y., van der Giezen, M., Tielens, A. G., and Martin, W. F. (2012) Biochemistry and evolution of anaerobic energy metabolism in eukaryotes. Microbiol Mol Biol Rev 76, 444-495

5. Baernstein, H. D. (1963) A review of electron transport mechanisms in parasitic protozoa. $J$ Parasitol 49, 12-21

6. Brown, D. M., Upcroft, J. A., Edwards, M. R., and Upcroft, P. (1998) Anaerobic bacterial metabolism in the ancient eukaryote Giardia duodenalis. Int J Parasitol 28, 149-164

7. Wallen, J. R., Mallett, T. C., Okuno, T., Parsonage, D., Sakai, H., Tsukihara, T., and Claiborne, A. (2015) Structural Analysis of Streptococcus pyogenes NADH Oxidase: Conformational Dynamics Involved in Formation of the C(4a)-Peroxyflavin Intermediate. Biochemistry 54, 68156829

8. Ross, R. P., and Claiborne, A. (1992) Molecular cloning and analysis of the gene encoding the $\mathrm{NADH}$ oxidase from Streptococcus faecalis $10 \mathrm{C} 1$. Comparison with NADH peroxidase and the flavoprotein disulfide reductases. $J$ Mol Biol 227, 658-671

9. Ojha, S., Meng, E. C., and Babbitt, P. C. (2007) Evolution of function in the "two dinucleotide binding domains" flavoproteins. PLoS Comput Biol 3, e121

10. Lountos, G. T., Jiang, R., Wellborn, W. B., Thaler, T. L., Bommarius, A. S., and Orville, A. M. (2006) The crystal structure of NAD(P)H oxidase from Lactobacillus sanfranciscensis: insights into the conversion of $\mathrm{O} 2$ into two water molecules by the flavoenzyme. Biochemistry 45, 96489659

11. Titov, D. V., Cracan, V., Goodman, R. P., Peng, J., Grabarek, Z., and Mootha, V. K. (2016) Complementation of mitochondrial electron transport chain by manipulation of the NAD+/NADH ratio. Science 352, 231-235 
12. Goncalves, V. L., Vicente, J. B., Pinto, L., Romao, C. V., Frazao, C., Sarti, P., Giuffre, A., and Teixeira, M. (2014) Flavodiiron oxygen reductase from Entamoeba histolytica: modulation of substrate preference by tyrosine 271 and lysine 53. J Biol Chem 289, 28260-28270

13. Silaghi-Dumitrescu, R., Coulter, E. D., Das, A., Ljungdahl, L. G., Jameson, G. N., Huynh, B. H., and Kurtz, D. M., Jr. (2003) A flavodiiron protein and high molecular weight rubredoxin from Moorella thermoacetica with nitric oxide reductase activity. Biochemistry 42, 2806-2815

14. Silaghi-Dumitrescu, R., Kurtz, D. M., Jr., Ljungdahl, L. G., and Lanzilotta, W. N. (2005) X-ray crystal structures of Moorella thermoacetica FprA. Novel diiron site structure and mechanistic insights into a scavenging nitric oxide reductase. Biochemistry 44, 6492-6501

15. Vicente, J. B., Carrondo, M. A., Teixeira, M., and Frazao, C. (2008) Structural studies on flavodiiron proteins. Methods in enzymology 437, 3-19

16. Vicente, J. B., Justino, M. C., Goncalves, V. L., Saraiva, L. M., and Teixeira, M. (2008) Biochemical, spectroscopic, and thermodynamic properties of flavodiiron proteins. Methods in enzymology 437, 21-45

17. Frazao, C., Silva, G., Gomes, C. M., Matias, P., Coelho, R., Sieker, L., Macedo, S., Liu, M. Y., Oliveira, S., Teixeira, M., Xavier, A. V., Rodrigues-Pousada, C., Carrondo, M. A., and Le Gall, J. (2000) Structure of a dioxygen reduction enzyme from Desulfovibrio gigas. Nat Struct Biol 7, 1041-1045

18. Smutna, T., Goncalves, V. L., Saraiva, L. M., Tachezy, J., Teixeira, M., and Hrdy, I. (2009) Flavodiiron protein from Trichomonas vaginalis hydrogenosomes: the terminal oxygen reductase. Eukaryotic cell 8, 47-55

19. Di Matteo, A., Scandurra, F. M., Testa, F., Forte, E., Sarti, P., Brunori, M., and Giuffre, A. (2008) The O2-scavenging flavodiiron protein in the human parasite Giardia intestinalis. J Biol Chem 283, 4061-4068

20. Vicente, J. B., Tran, V., Pinto, L., Teixeira, M., and Singh, U. (2012) A detoxifying oxygen reductase in the anaerobic protozoan Entamoeba histolytica. Eukaryotic cell 11, 1112-1118

21. Page, C. C., Moser, C. C., Chen, X., and Dutton, P. L. (1999) Natural engineering principles of electron tunnelling in biological oxidation-reduction. Nature 402, 47-52

22. Jenney, F. E., Jr., and Adams, M. W. (2001) Rubredoxin from Pyrococcus furiosus. Methods in enzymology 334, 45-55

23. Kawasaki, S., Sakai, Y., Takahashi, T., Suzuki, I., and Niimura, Y. (2009) O2 and reactive oxygen species detoxification complex, composed of O2-responsive NADH:rubredoxin oxidoreductase-flavoprotein A2-desulfoferrodoxin operon enzymes, rubperoxin, and rubredoxin, in Clostridium acetobutylicum. Appl Environ Microbiol 75, 1021-1029

24. Lee, W. Y., Brune, D. C., LoBrutto, R., and Blankenship, R. E. (1995) Isolation, characterization, and primary structure of rubredoxin from the photosynthetic bacterium, Heliobacillus mobilis. Arch Biochem Biophys 318, 80-88

25. LeGall, J., Prickril, B. C., Moura, I., Xavier, A. V., Moura, J. J., and Huynh, B. H. (1988) Isolation and characterization of rubrerythrin, a non-heme iron protein from Desulfovibrio vulgaris that contains rubredoxin centers and a hemerythrin-like binuclear iron cluster. Biochemistry 27, 1636-1642

26. Lovenberg, W., and Sobel, B. E. (1965) Rubredoxin: a new electron transfer protein from Clostridium pasteurianum. Proc Natl Acad Sci U S A 54, 193-199

27. Folgosa, F., Martins, M. C., and Teixeira, M. (2018) Diversity and complexity of flavodiiron $\mathrm{NO} / \mathrm{O} 2$ reductases. FEMS Microbiol Lett 365

28. Romao, C. V., Vicente, J. B., Borges, P. T., Frazao, C., and Teixeira, M. (2016) The dual function of flavodiiron proteins: oxygen and/or nitric oxide reductases. J Biol Inorg Chem 21, 39-52

29. Saraiva, L. M., Vicente, J. B., and Teixeira, M. (2004) The role of the flavodiiron proteins in microbial nitric oxide detoxification. Adv Microb Physiol 49, 77-129

30. Tanabe, M. (1979) Trichomonas vaginalis: NADH oxidase activity. Exp Parasitol 48, 135-143 
31. Linstead, D. J., and Bradley, S. (1988) The purification and properties of two soluble reduced nicotinamide: acceptor oxidoreductases from Trichomonas vaginalis. Molecular and biochemical parasitology 27, 125-133

32. Brown, D. M., Upcroft, J. A., and Upcroft, P. (1996) A H2O-producing NADH oxidase from the protozoan parasite Giardia duodenalis. European journal of biochemistry / FEBS 241, 155-161

33. Castillo-Villanueva, A., Mendez, S. T., Torres-Arroyo, A., Reyes-Vivas, H., and Oria-Hernandez, J. (2016) Cloning, Expression and Characterization of Recombinant, NADH Oxidase from Giardia lamblia. Protein J 35, 24-33

34. Nixon, J. E., Wang, A., Field, J., Morrison, H. G., McArthur, A. G., Sogin, M. L., Loftus, B. J., and Samuelson, J. (2002) Evidence for lateral transfer of genes encoding ferredoxins, nitroreductases, NADH oxidase, and alcohol dehydrogenase 3 from anaerobic prokaryotes to Giardia lamblia and Entamoeba histolytica. Eukaryotic cell 1, 181-190

35. Vicente, J. B., Testa, F., Mastronicola, D., Forte, E., Sarti, P., Teixeira, M., and Giuffre, A. (2009) Redox properties of the oxygen-detoxifying flavodiiron protein from the human parasite Giardia intestinalis. Arch Biochem Biophys 488, 9-13

36. Ahmed, S. A., and Claiborne, A. (1989) The streptococcal flavoprotein NADH oxidase. II. Interactions of pyridine nucleotides with reduced and oxidized enzyme forms. J Biol Chem 264, 19863-19870

37. Hagelueken, G., Wiehlmann, L., Adams, T. M., Kolmar, H., Heinz, D. W., Tummler, B., and Schubert, W. D. (2007) Crystal structure of the electron transfer complex rubredoxin rubredoxin reductase of Pseudomonas aeruginosa. Proc Natl Acad Sci U S A 104, 12276-12281

38. Folgosa, F., Martins, M. C., and Teixeira, M. (2018) The multidomain flavodiiron protein from Clostridium difficile 630 is an NADH:oxygen oxidoreductase. Sci Rep 8, 10164

39. Kelley, L. A., Mezulis, S., Yates, C. M., Wass, M. N., and Sternberg, M. J. (2015) The Phyre2 web portal for protein modeling, prediction and analysis. Nat Protoc 10, 845-858

40. Hampl, V., Hug, L., Leigh, J. W., Dacks, J. B., Lang, B. F., Simpson, A. G., and Roger, A. J. (2009) Phylogenomic analyses support the monophyly of Excavata and resolve relationships among eukaryotic "supergroups". Proc Natl Acad Sci U S A 106, 3859-3864

41. Gao, H., Li, J., Sivakumar, D., Kim, T. S., Patel, S. K. S., Kalia, V. C., Kim, I. W., Zhang, Y. W., and Lee, J. K. (2019) NADH oxidase from Lactobacillus reuteri: A versatile enzyme for oxidized cofactor regeneration. Int J Biol Macromol 123, 629-636

42. Higuchi, M. (1992) Reduced nicotinamide adenine dinucleotide oxidase involvement in defense against oxygen toxicity of Streptococcus mutans. Oral Microbiol Immunol 7, 309-314

43. Lamien-Meda, A., and Leitsch, D. (2020) Identification of the NADH-oxidase gene in Trichomonas vaginalis. Parasitol Res 119, 683-686

44. Erickson, H. P. (2009) Size and Shape of Protein Molecules at the Nanometer Level Determined by Sedimentation, Gel Filtration, and Electron Microscopy. Biological Procedures Online 11, 32

45. Warrenfeltz, S., Basenko, E. Y., Crouch, K., Harb, O. S., Kissinger, J. C., Roos, D. S., Shanmugasundram, A., and Silva-Franco, F. (2018) EuPathDB: The Eukaryotic Pathogen Genomics Database Resource. Methods Mol Biol 1757, 69-113

46. Aliverti, A., Curti, B., and Vanoni, M. A. (1999) Identifying and quantitating FAD and FMN in simple and in iron-sulfur-containing flavoproteins. Methods Mol Biol 131, 9-23

47. Mastronarde, D. N. (2005) Automated electron microscope tomography using robust prediction of specimen movements. J Struct Biol 152, 36-51

48. Punjani, A., Rubinstein, J. L., Fleet, D. J., and Brubaker, M. A. (2017) cryoSPARC: algorithms for rapid unsupervised cryo-EM structure determination. Nat Methods 14, 290-296

49. Zivanov, J., Nakane, T., Forsberg, B. O., Kimanius, D., Hagen, W. J., Lindahl, E., and Scheres, S. H. (2018) New tools for automated high-resolution cryo-EM structure determination in RELION3. Elife 7 
50. Bepler, T., Morin, A., Rapp, M., Brasch, J., Shapiro, L., Noble, A. J., and Berger, B. (2019) Positive-unlabeled convolutional neural networks for particle picking in cryo-electron micrographs. Nat Methods 16, 1153-1160

51. Zheng, S. Q., Palovcak, E., Armache, J. P., Verba, K. A., Cheng, Y., and Agard, D. A. (2017) MotionCor2: anisotropic correction of beam-induced motion for improved cryo-electron microscopy. Nat Methods 14, 331-332

52. Rohou, A., and Grigorieff, N. (2015) CTFFIND4: Fast and accurate defocus estimation from electron micrographs. $J$ Struct Biol 192, 216-221

53. Herzik, M. A., Jr., Wu, M., and Lander, G. C. (2019) High-resolution structure determination of sub-100 kDa complexes using conventional cryo-EM. Nat Commun 10, 1032

54. Kucukelbir, A., Sigworth, F. J., and Tagare, H. D. (2014) Quantifying the local resolution of cryo-EM density maps. Nat Methods 11, 63-65

55. Pettersen, E. F., Goddard, T. D., Huang, C. C., Couch, G. S., Greenblatt, D. M., Meng, E. C., and Ferrin, T. E. (2004) UCSF Chimera--a visualization system for exploratory research and analysis. J Comput Chem 25, 1605-1612

56. Stein, A., and Kortemme, T. (2013) Improvements to robotics-inspired conformational sampling in rosetta. PLoS One 8, e63090

57. Afonine, P. V., Poon, B. K., Read, R. J., Sobolev, O. V., Terwilliger, T. C., Urzhumtsev, A., and Adams, P. D. (2018) Real-space refinement in PHENIX for cryo-EM and crystallography. Acta Crystallogr D Struct Biol 74, 531-544

58. The PyMOL Molecular Graphics System, Version 2.0 Schrödinger, LLC.

\author{
Abbreviations and nomenclature \\ ETC: electron transport chain; ET: electron transport; FDP: flavodiiron protein; NROR: NADH:rubredoxin \\ oxidoreductase; Rb: rubredoxin;
}

Unpublished observations and personal communications

None 


\section{Tables}

Table 1.

FAD and FMN quantification.

\begin{tabular}{lcc}
\hline Protein & $\begin{array}{c}\text { FAD per protein } \\
\text { monomer }\end{array}$ & $\begin{array}{c}\text { FMN per protein } \\
\text { monomer }\end{array}$ \\
\hline $\boldsymbol{T} v$ FDPF1 & $1.02 \pm 0.05$ & $0.010 \pm 0.004$ \\
$\boldsymbol{T} v$ FDPF1* & $1.12 \pm 0.08$ & $0.010 \pm 0.004$ \\
$\boldsymbol{T} v$ FDPF2 & $0.32 \pm 0.05$ & $0.009 \pm 0.002$ \\
$\boldsymbol{T} \boldsymbol{v}$ FDPF3 & $1.00 \pm 0.12$ & $0.090 \pm 0.006$ \\
holoT $\boldsymbol{v}$ FDPF3 & $0.94 \pm 0.09$ & $0.92 \pm 0.09$ \\
\hline
\end{tabular}

Cofactors were quantified using LC-MS as described under "Experimental procedures". Stoichiometry was calculated per protein monomers. Data represents $(n=4-6)$ independent experiments \pm s.d. "Holo" refers to highly active $T v$ FDPF3 fully loaded with FAD and FMN cofactors. * $0.3 \mathrm{mM}$ FMN was added to the lysis buffer during purification. 


\section{Table 2.}

Steady-state kinetic parameters of the enzymatic reactions catalyzed by $T v$ FDPF1-3 and holoTvFDPF3.

\begin{tabular}{|c|c|c|c|c|c|}
\hline Protein & Substrate & $\begin{array}{c}K_{m} \\
(\mu \mathrm{M})\end{array}$ & $\begin{array}{c}V_{\max } \\
\left(\mu \mathrm{mol} \mathrm{min}^{-1}\right. \\
\left.\mathrm{mg}^{-1}\right)\end{array}$ & $\begin{array}{l}k_{\text {cat }} \\
\left(\mathrm{s}^{-1}\right)\end{array}$ & $\begin{array}{c}k_{\text {cat }} / K_{m} \\
\left(s^{-1} M^{-1}\right)\end{array}$ \\
\hline \multirow[t]{2}{*}{$T v F D P F 1$} & $\mathrm{NADH}$ & $2.9 \pm 0.8$ & $4.9 \pm 1.2$ & $7.8 \pm 1.9$ & $(2.6 \pm 0.9) \times 10^{6}$ \\
\hline & NADPH & $1475 \pm 486$ & $4.9 \pm 2.1$ & $7.8 \pm 3.3$ & $(5.2 \pm 2.8) \times 10^{3}$ \\
\hline \multirow[t]{2}{*}{$T v F D P F 2$} & NADH & $18 \pm 2$ & $6.1 \pm 0.2$ & $9.6 \pm 0.3$ & $(5.3 \pm 0.6) \times 10^{5}$ \\
\hline & NADPH & $1171 \pm 208$ & $1.1 \pm 0.2$ & $1.7 \pm 0.3$ & $(1.4 \pm 0.3) \times 10^{3}$ \\
\hline \multirow[t]{2}{*}{$T v$ FDPF3 } & NADH & $40 \pm 8$ & $12 \pm 1$ & $19 \pm 2$ & $(4.8 \pm 1.0) \times 10^{5}$ \\
\hline & NADPH & $399 \pm 8$ & $4.0 \pm 0.1$ & $6.4 \pm 0.2$ & $(1.6 \pm 0.0) \times 10^{4}$ \\
\hline \multirow[t]{2}{*}{ holoTvFDPF3 } & NADH & $56 \pm 2$ & $291 \pm 26$ & $466 \pm 42$ & $(8.3 \pm 0.8) \times 10^{6}$ \\
\hline & NADPH & $427 \pm 160$ & $11 \pm 4$ & $18 \pm 6$ & $(4.1 \pm 2.1) \times 10^{4}$ \\
\hline
\end{tabular}

Activities were measured as described under "Experimental procedures". Kinetic parameters represent the average of $(\mathrm{n}=4-8)$ independent experiments \pm s.d. $k_{\text {cat }}$ values were calculated per protein dimer. 
Figures and figure legends

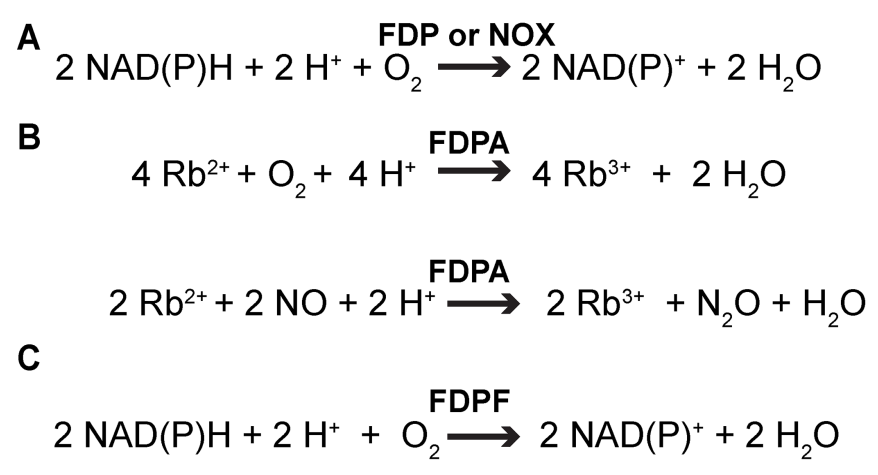

Figure 1. Reactions catalyzed by Class A and Class F Flavodiiron Proteins (FDPA and FDPF) as well as by a $\mathrm{H}_{2} \mathrm{O}$-forming $\mathbf{N A D H}$ oxidase (NOX). (a) The net $\mathrm{H}_{2} \mathrm{O}$-forming $\mathrm{NAD}(\mathrm{P}) \mathrm{H}$ oxidase reaction catalyzed by a multicomponent flavodiiron protein (FDP)-based system or a $\mathrm{H}_{2} \mathrm{O}$-forming NADH oxidase (NOX). (b) In the simplest configuration, Class A FDP (FDPA) receives electrons from reduced rubredoxin $(\mathrm{Rb})$. Some FDPAs can also reduce nitric oxide to nitrous oxide and water, respectively. (c) Class F FDP (FDPF) represents a natural fusion, where the FDP core protein is fused with both rubredoxin (Rb) and NADH:rubredoxin oxidoreductase (NROR) redox partners. 

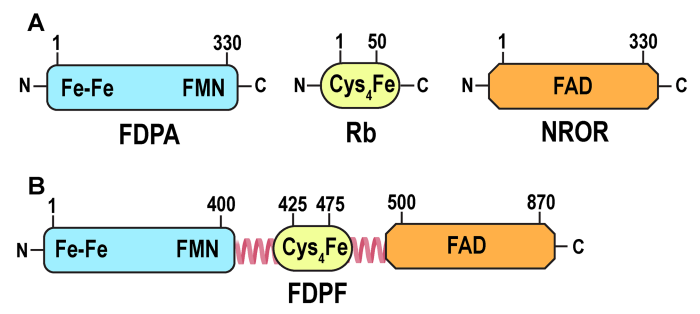

Figure 2. Domain organization of Class $A$ and Class $F$ flavodiiron proteins (FDPA and FDPF). (a) Class A flavodiiron protein (FDPA) contains the diiron center (Fe-Fe) and a flavin cofactor (FMN). Two additional redox partners of a "stand-alone" FDPA are: (i) rubredoxin (Rb) and (ii) NADH:rubredoxin oxidoreductase (NROR) (see Fig. 1). The $\mathrm{Fe}(\mathrm{SCys})_{4}$ center of $\mathrm{Rb}$ is formed by a central iron which is coordinated by four cysteines. The redox center of NROR contains a flavin redox cofactor (FAD). (b) Class F FDP (FDPF) represents a natural fusion between the N-terminal FDP core, Rb-like domain, and NRORlike domain. Loops connecting the middle rubredoxin-like domain with the N-terminal FDP core and the NROR domain are shown. 

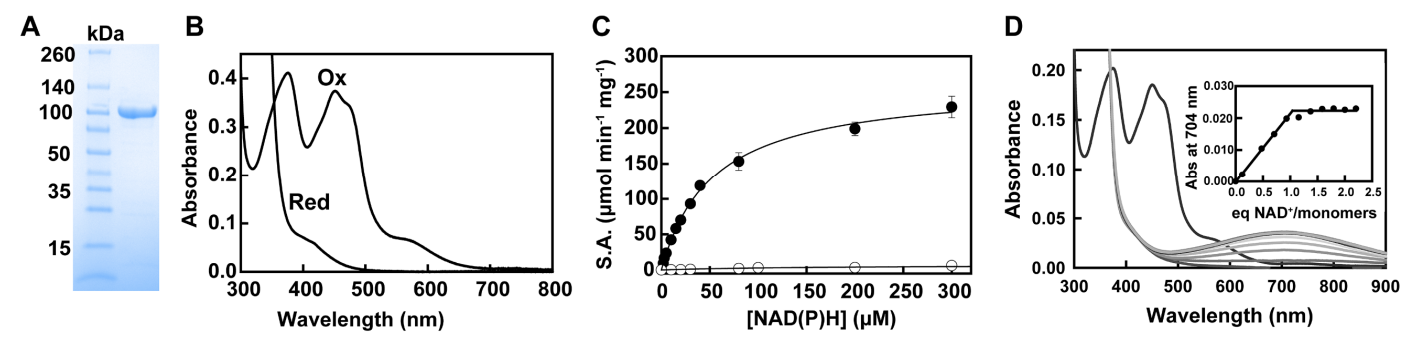

Figure 3. Biochemical and spectroscopic properties of highly active holoTvFDPF3. (a) Purified holoTvFDPF3 (10 $\mu$ g per lane). (b) UV-visible spectra of holoTvFDPF3 as purified. Protein was in buffer $\mathrm{E}$ at $20 \mu \mathrm{M}$ (calculated based on the molecular weight of a monomer) as purified (Ox.) and after addition of $1 \mathrm{mM}$ of sodium dithionite (Red.) under aerobic conditions. (c) Michaelis-Menten analysis of the oxidase activity of holoTvFDPF3 as described under "Experimental procedures" with NADH (filled circles) and NADPH (open circles). All kinetic parameters are summarized in Table 2. (d) Spectral titration of dithionite-reduced holo $T \nu \mathrm{FDPF} 3$ with $\mathrm{NAD}^{+}$. Under anaerobic conditions holoT $\nu \mathrm{FDPF} 3(8.18 \mu \mathrm{M}$ of monomers) was reduced with $1 \mathrm{mM}$ dithionite before different amounts of NAD ${ }^{+}$were added. Spectra shown represent the starting oxidized enzyme, the dithionite-reduced enzyme and the reduced enzyme plus 0.12 - 2.2 equivalents of $\mathrm{NAD}^{+} /$monomer. The inset shows the absorbance change at $704 \mathrm{~nm}$ versus equivalents of $\mathrm{NAD}^{+} /$monomer. 
A

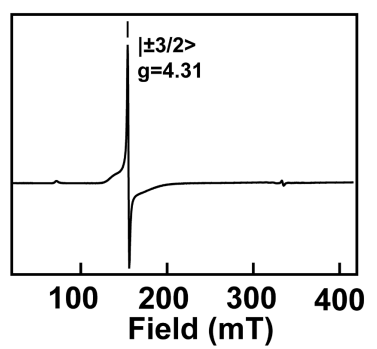

B

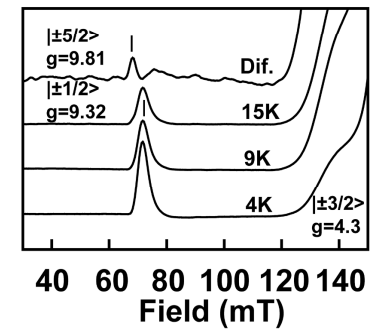

C

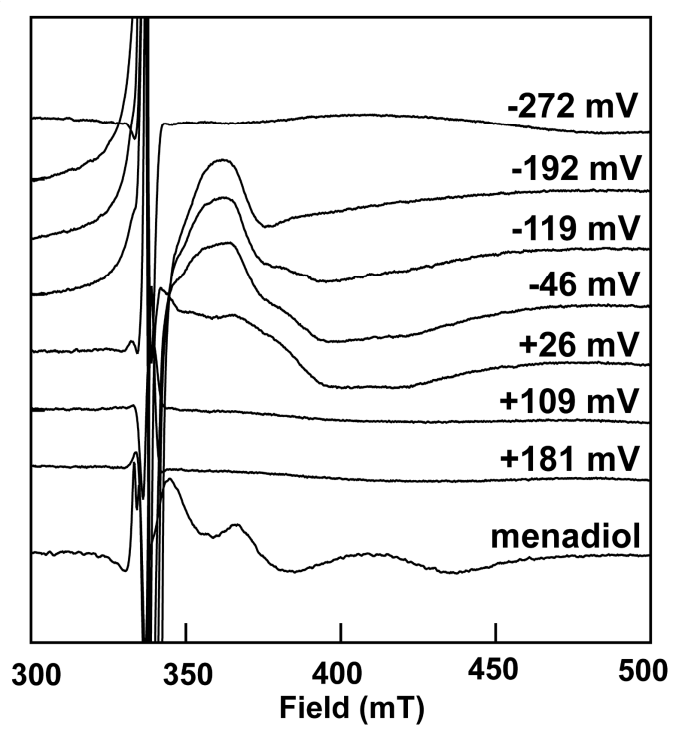

Figure 4. EPR analysis of holoTvFDPF3. Spectra of the ferric rubredoxin site in the as isolated enzyme at $15 \mathrm{~K}$ (a), or at indicated temperatures (including a $15 \mathrm{~K}$ minus $9 \mathrm{~K}$ difference spectrum) (b). (c) Spectra recorded at $7.5 \mathrm{~K}$ for samples poised at the indicated redox potentials $\left(\mathrm{vs} . \mathrm{H}_{2} / \mathrm{H}^{+}\right.$at $\mathrm{pH} 7.5$ ) or poised at $+115 \mathrm{mV}$ and treated with $50 \mu \mathrm{M}$ menadiol (all spectra were recorded at X-band, microwave frequency $9.358 \pm 0.003 \mathrm{GHz}$; modulation amplitude, $1.5 \mathrm{mT} ; 2 \mathrm{~mW}$ microwave power; modulation frequency 100 $\mathrm{kHz}$ ) as described under Experimental procedures. 


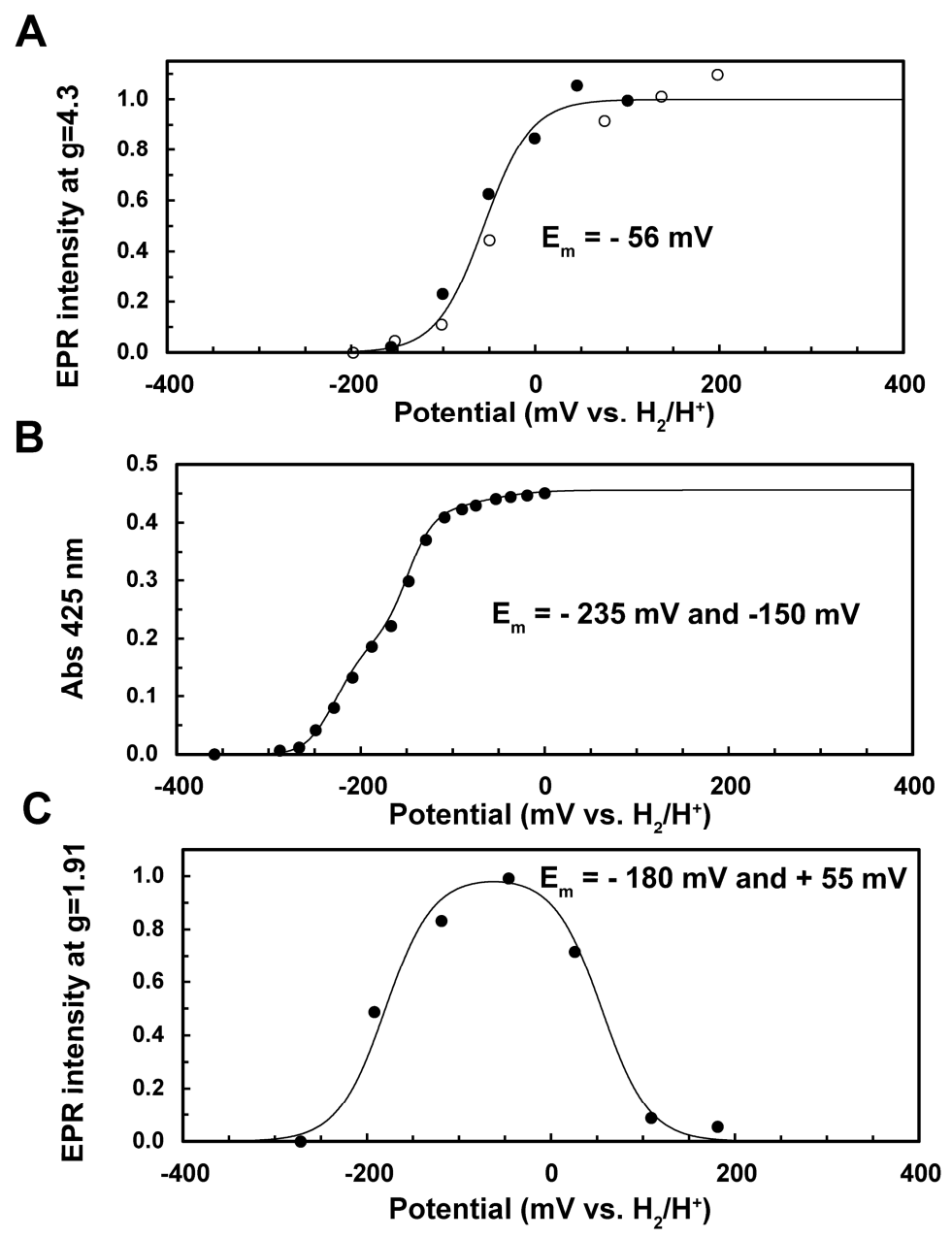

Figure 5. Determination of redox potentials of cofactors in holoTvFDPF3. (a) Intensities of the $g=4.3$ EPR signal (recorded at $77 \mathrm{~K}$ ) of the rubredoxin site in frozen samples upon dye-mediated redox titrations (open and closed symbols refer two separate titrations). (b) Intensity of the mixed-valence EPR signal (amplitude at $g=1.91$ ) of the dinuclear iron center upon dye-mediated redox titration. EPR conditions as in Fig. 4. (c) Absorbance at $425 \mathrm{~nm}$ (corrected for contribution of the mediator cocktail in a parallel titration) reporting mainly on the presence of the oxidized flavin cofactors at room temperature. Fits to the (sum of) Nernst equation(s) are shown: rubredoxin site $\mathrm{E}_{\mathrm{m}}=-56 \mathrm{mV}, \mathrm{n}=1$ (a), FAD, $\mathrm{E}_{\mathrm{m}}=-235(\mathrm{n}=1)$ and $-235 \mathrm{mV}$ $(\mathrm{n}=1), F M N, \mathrm{E}_{\mathrm{m}}=-190(\mathrm{n}=1)$ and $-110 \mathrm{mV}(\mathrm{n}=1)$, flavins each with a 0.215 absorbance plus an absorbance of 0.026 for the rubredoxin site $\left(E_{m}=-56 \mathrm{mV}, \mathrm{n}=1\right)$ in (c). In (b) the curve for $E_{m}=-180(n=1)$ and $+55 \mathrm{mV}$ $(\mathrm{n}=1)$ indicates the approximate redox potentials. 

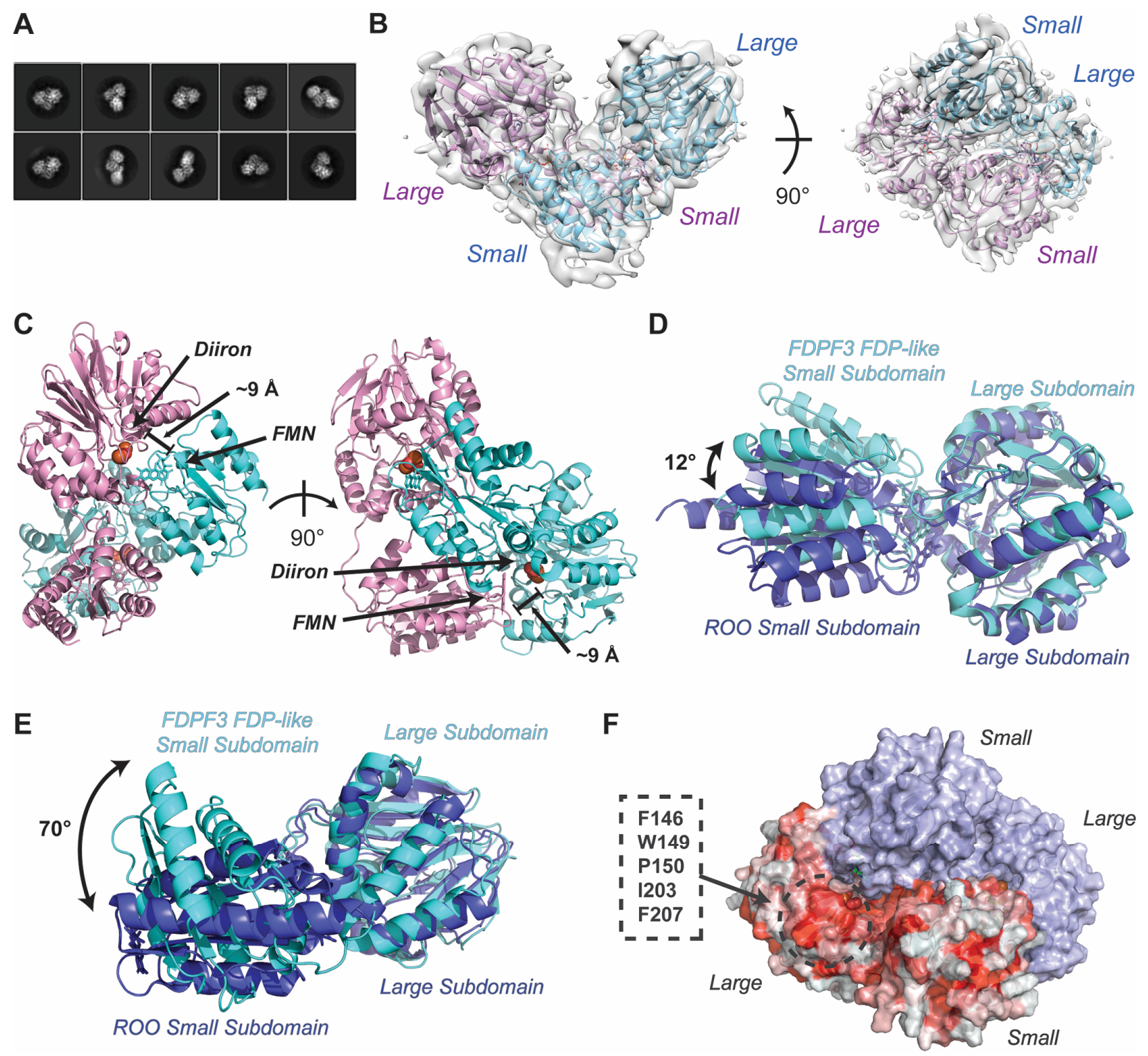

Figure 6. Cryo-EM structure of the FDP-like core of the full-length TvFDPF3. (a) Representative 2D class averaged images of holoTvFDPF3 particles. (b) Homology models docked into the reconstructed single-particle cryo-EM C2-symmetric map. (c) Distance between diiron center and FMN in the holoTvFDPF3 FDP-like domain dimer. (d) Aligned overlay of the holoTvFDPF3 FDP-like domain redox center with the redox center in ROO from D. gigas (PDB 1E5D) (17). (e) Aligned overlay of one holoTvFDPF3 FDP-like domain subunit with a subunit of ROO from D. gigas, as above. (f) Surface representation of the dimerized FDP-like domain model with one subunit colored by hydrophobicity. A hydrophobic patch that is concealed in the D. gigas ROO head-to-tail dimer is highlighted. 


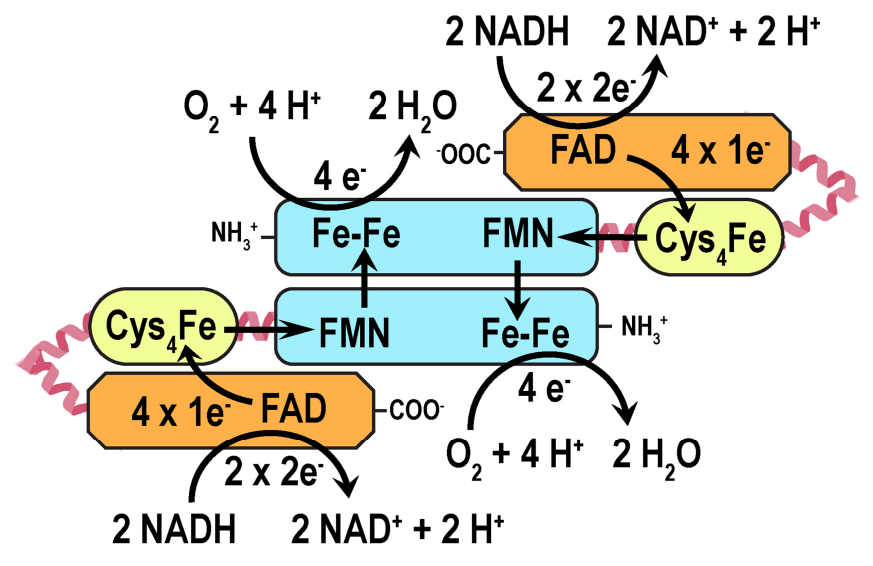

Figure 7. Proposed electron transport (ET) pathway within the dimer of holoTvFDPF3. Color scheme of FDP, Rb and NROR domains is the same as in Fig. 2. 